\title{
THE ATIYAH-SINGER INDEX THEOREM
}

\author{
DANIEL S. FREED
}

In memory of Michael Atiyah

\begin{abstract}
The Atiyah-Singer index theorem, a landmark achievement of the early 1960s, brings together ideas in analysis, geometry, and topology. We recount some antecedents and motivations, various forms of the theorem, and some of its implications, which extend to the present.
\end{abstract}

\section{Contents}

1. Introduction

2. Antecedents and motivations from algebraic geometry and topology

3. Antecedents in analysis

4. The index theorem and proofs

5. Variations on the theme

6. Heat equation proof

7. Geometric invariants of Dirac operators

8. Anomalies and index theory

About the author

References

\section{INTRODUCTION}

Consider the Riemann sphere $\mathbb{C P}^{1}=\mathbb{C} \cup\{\infty\}$. Let $\left\{z_{i}\right\}_{i=1}^{N} \subset \mathbb{C P}^{1}$ be a finite set, and to each $i \in\{1, \ldots, N\}$ suppose a nonzero integer $n_{i}$ is given. A classical problem asks for a meromorphic function $f$ with a zero or pole at each $z_{i}$. If $n_{i}>0$, then $z_{i}$ is a zero of multiplicity $n_{i}$; if $n_{i}<0$, then $z_{i}$ is a pole of order $\left|n_{i}\right|$. The solution is straightforward. Namely, $f(z)=c \prod\left(z-z_{i}\right)^{n_{i}}$ is a rational function, unique up to the constant $c \in \mathbb{C} \backslash\{0\}$. In other words, now allowing $f \equiv 0$, the solutions form a one-dimensional complex vector space. If we replace $\mathbb{C P}^{1}$ by a closed Riemann surface of positive genus, then there is an obstruction to existence of a meromorphic function with specified zeros and poles. For example, an elliptic curve can be realized as a quotient $E=\mathbb{C} /(\mathbb{Z}+\mathbb{Z} \tau)$ of the complex line by the full lattice generated by $1, \tau$ for some $\tau \in \mathbb{C}$ with $\operatorname{Im} \tau>0$. A meromorphic function on $E$ lifts to a doubly periodic function on $\mathbb{C}$, and the single constraint on the zeros and poles of a meromorphic function is $\sum n_{i} z_{i} \in \mathbb{Z}+\mathbb{Z} \tau$. Proceeding from $E$ to

Received by the editors June 10, 2021.

2020 Mathematics Subject Classification. Primary 58J20, 58J28, 58J52, 19K56, 81T50.

This material is based upon work supported by the National Science Foundation under Grant Number DMS-2005286. 
a general closed Riemann surface $X$, we encounter more constraints. In fact, the constraints form a vector space whose dimension is the genus of $X$, a topological invariant. Meromorphic functions are solutions to the Cauchy-Riemann equation, a linear elliptic partial differential equation. The solutions and obstructions to this elliptic PDE are "counted" via topology. In a more general form, this is the classical Riemann-Roch theorem.

The Atiyah-Singer index theorem, formulated and proved in 1962-63, is a vast generalization to arbitrary elliptic operators on compact manifolds of arbitrary dimension. The Fredholm index in question is the dimension of the kernel minus the dimension of the cokernel of a linear elliptic operator. The Atiyah-Singer theorem computes the index in terms of topological invariants of the operator and topological invariants of the underlying manifold. The theorem weaves together concepts and results in algebraic topology, algebraic geometry, differential geometry, and linear analysis; its ramifications go far beyond, in number theory, representation theory, operator algebras, nonlinear analysis, and theoretical physics. Furthermore, index theory itself is a sprawling enterprise. The basic Atiyah-Singer theorem spawned numerous generalizations and novel pathways. This paper - a tribute to Michael Atiyah - naturally focuses on aspects of his work and his influence. Even thus restricted, we can only skim the surface of this rich story.

There are antecedents of the index theorem from algebraic geometry and topology on the one hand, and from analysis on the other. We discuss these in turn in 92 and 93 . The basic Atiyah-Singer theorem is the subject of 94 . The first proof is based on cobordism and in broad outline follows Hirzebruch's proofs of his signature and Riemann-Roch theorems. The second proof is based on $K$-theory; it is inspired by Grothendieck's Riemann-Roch theorem. In $\$ 5$ we take up some of the extensions and variations of the basic theorem. These include an equivariant index theorem, the index theorem for parametrized families of operators, the index theorem for manifolds with boundary, and a few more. At this point our exposition makes a transition from global topological invariants of general linear elliptic operators to local geometric invariants of Dirac operators. Heat equation methods are the subject of 86 , the first application being a local index theorem. New geometric invariants of Dirac operators appear in 87 . In 88 we turn to physics, which was Atiyah's focus after the mid-1980s and which provided an unanticipated playground for the circle of ideas surrounding the index theorem. We focus on anomalies in quantum theory, a subject to which Atiyah and Singer made an early contribution.

Each section of this paper has more introductory material, which we recommend even to the casual reader. Also, a lecture based on this paper may be viewed at [F1].

Michael had great mathematical and personal charisma. His writings capture his vibrancy, as did his lectures, some of which are available online. He wrote many wonderful expository articles about the index theorem, especially of the early period; you will enjoy perusing them.

I warmly thank Simon Donaldson, Charlie Reid, and Graeme Segal for their careful reading of and comments on an earlier version.

\section{Antecedents And motivations From Algebraic GeOMEtry AND TOPOLOGY}

Enumerative problems in algebraic geometry often lead to integers that have a topological interpretation. A classical example is the Riemann-Roch formula, 
which is our starting point in 92.1 The higher-dimensional generalization was taken up by Fritz Hirzebruch in the early 1950s, as we recount in \$2.2, A few years later Alexander Grothendieck extended Hirzebruch's theorem to a relative version, that is, to proper maps of complex manifolds. In the process he introduced $K$ theory for sheaves. His ideas, briefly presented in $\$ 2.3$, play a fundamental role in variations of the Atiyah-Singer index theorem a decade later. More immediately, as Graeme Segal writes in this volume [Seg4, Atiyah and Hirzebruch transported Grothendieck's $K$-theory over to algebraic topology. Raoul Bott's computation of the stable homotopy groups of Lie groups, which took place during the same period as Hirzebruch's and Grothendieck's work on the Riemann-Roch theorem, is the cornerstone of their theory. Crucial for the index theorem are the resulting integrality theorems, of which we mention a few in 2.4. This led to a questionWhy is the $\hat{A}$-genus an integer for a spin manifold?-which in early 1962 was the immediate catalyst for Atiyah and Singer's collaboration.

2.1. The Riemann-Roch theorem. Let $X$ be a smooth projective curve over $\mathbb{C}$, i.e., a one-dimensional closed complex submanifold of a complex projective space. A divisor $D$ is a finite set of points on $X$ with an integer $\operatorname{ord}_{x}(D)$ attached to each point $x \in D$. A divisor determines a holomorphic line bundle on $X$; let $\mathcal{L}(D)$ denote the space of holomorphic sections of this bundle. We can describe $\mathcal{L}(D)$ as the space of meromorphic functions on $X$ which have a pole of order $\leq \operatorname{ord}_{x}(D)$ at each $x \in X$. A basic problem in the theory of curves is: Compute the dimension of $\mathcal{L}(D)$. While this is quite difficult in general, there is a topological formula for $\operatorname{dim} \mathcal{L}(D)-\operatorname{dim} \mathcal{L}(K-D)$, where $K$ is a canonical divisor of $X$. (The zero set of a holomorphic 1-form, weighted by the orders of the zeros, is a canonical divisor.)

Theorem 2.1 (Riemann-Roch). Let $X$ be a smooth projective curve, and let $D$ be a divisor on $X$. Then

$$
\operatorname{dim} \mathcal{L}(D)-\operatorname{dim} \mathcal{L}(K-D)=\operatorname{deg}(D)-g+1 .
$$

Here $g$ is the genus of the curve $X$, its fundamental topological invariant, which is defined to be $\frac{1}{2} \operatorname{rank} H^{1}(X ; \mathbb{Z})$. Also, $\operatorname{deg}(D)=\sum \operatorname{ord}_{x}(D)$ is the sum of the integers which define the divisor $D$. If $\operatorname{deg}(D)>2 g-2$, it can be shown that $\mathcal{L}(K-D)=0$, so that in that case (2.2) provides a complete solution to the problem of computing $\operatorname{dim} \mathcal{L}(D)$. Theorem 2.1 is the classical Riemann-Roch 1 formula. The Atiyah-Singer index theorem is a vast generalization of (2.2), as we will see.

Let us immediately note one consequence of the Riemann-Roch formula. Take $D=\mathcal{O}$ to be the trivial divisor consisting of no points. Then $\mathcal{L}(\mathcal{O})$ is the space of constant functions and $\mathcal{L}(K)$ is the space of holomorphic differentials. We deduce from (2.2) that the latter has dimension $g$. It follows that $g$ is an integer, i.e., rank $H^{1}(X ; \mathbb{Z})$ is even. Therefore, one-half the Euler number $\operatorname{Euler}(X)$ is an integer, our first example of an integrality theorem. The proof is noteworthy: $1-\operatorname{Euler}(X) / 2$ is an integer because it is the dimension of a vector space, namely $\mathcal{L}(K)$.

\footnotetext{
${ }^{1}$ Riemann Ri] proved the inequality $\operatorname{dim} \mathcal{L}(D) \geq \operatorname{deg}(D)-g+1$, and then Roch Ro proved the more precise (2.2). Sadly, Roch died of tuberculosis at the age of 26 , just months after the 39-year-old Riemann succumbed to tuberculosis.
} 
In the last decade of the $19^{\text {th }}$ century, Noether, Enriques, and Castelnuovo generalized the Riemann-Roch inequality and equality to algebraic surfaces; see (2.4) below.

2.2. Hirzebruch's Riemann-Roch and signature theorems. We skip far ahead to the years 1945-1954 and the work of young Hirzebruch, based on two important developments in geometry. The first, initiated by Leray, is the theory of sheaves. The second are the results in Thom's thesis, particularly those concerning bordism 2 groups of smooth manifolds. We state two of Hirzebruch's main results, which are recounted in [H1].

Let $X$ be a nonsingular projective variety of complex dimension $n$, and let $V \rightarrow$ $X$ be a holomorphic vector bundle. (In our discussion of curves we used divisors; recall that a divisor determines a holomorphic line bundle, which makes the link to our formulation here.) Then the cohomology groups $H^{q}(X, V)$ are defined via sheaf theory: $H^{0}(X, V)$ is the vector space of holomorphic sections of $V \rightarrow X$, and $H^{q}(X, V)$ for $q \geq 1$ are derived from resolutions of the sheaf of holomorphic sections of $V \rightarrow X$. The cohomology groups are finite dimensional, which can be proved using the theory of elliptic differential operators and Dolbeault's theorem. (See $\S 3.13 .2$ ) The Euler characteristic is defined as the alternating sum

$$
\chi(X, V)=\sum_{q=0}^{n}(-1)^{q} \operatorname{dim} H^{q}(X, V) .
$$

As for the case $n=1$ of Riemann surfaces, one often wants to compute $\operatorname{dim} H^{0}(X, V)$, but in general $\operatorname{dim} H^{0}(X, V)$ depends on more than topological data. On the other hand, the Euler characteristic $\chi(X, V)$ does have a topological formula in terms of the Chern classes $c_{j}(X)$ and $c_{k}(V)$. The special case $\operatorname{dim} X=\operatorname{rank} V=1$ is the classical Riemann-Roch formula (2.2). For $X$ a smooth projective algebraic surface $(n=2)$ and $V \rightarrow X$ the trivial bundle of rank 1 , the result is commonly known as Noether's formula:

$$
\chi(X)=\frac{1}{12}\left(c_{1}^{2}(X)+c_{2}(X)\right)[X] .
$$

In (2.4) the Chern classes are evaluated on the fundamental class of $X$ given by the natural orientation. The presence of 12 in the denominator gives an integrality theorem for the Chern numbers of a projective surface.

The solution to the Riemann-Roch problem for all $X, V$ - that is, the computation of (2.3) - is one of Hirzebruch's signal achievements. Hirzebruch's formula is expressed in terms of the Todd polynomials and the Chern character. Suppose that the tangent bundle $T X=L_{1} \oplus \cdots \oplus L_{n}$ splits as a sum of line bundles, and set $y_{i}=c_{1}\left(L_{i}\right) \in H^{2}(X ; \mathbb{Z})$. Then the Todd class is

$$
\operatorname{Todd}(X)=\prod_{i=1}^{n} \frac{y_{i}}{1-e^{-y_{i}}} .
$$

\footnotetext{
${ }^{2}$ Thom, Hirzebruch, and many others use "cobordism" in place of "bordism"; Atiyah A10 clarified the relationship.
} 
This is a cohomology class of (mixed) even degree. Similarly, if $V=K_{1} \oplus \cdots \oplus K_{r}$ is a sum of line bundles, with $x_{i}=c_{1}\left(K_{i}\right)$, then the Chern character is

$$
\operatorname{ch}(V)=\sum_{i=1}^{r} e^{x_{i}} .
$$

The splitting principle in the theory of characteristic classes allows us to extend these definitions to $T X \rightarrow X$ and $V \rightarrow X$ which are not sums of line bundles.

Theorem 2.7 (Hirzebruch's Riemann-Roch theorem). Let $X$ be a projective complex manifold, and let $V \rightarrow X$ be a holomorphic vector bundle. Then

$$
\chi(X, V)=\operatorname{Todd}(X) \operatorname{ch}(V)[X] .
$$

Hirzebruch's second main theorem, which is a step in the proof of Theorem 2.7 is now called Hirzebruch's signature theorem. Let $X$ be a closed oriented real differentiable manifold of dimension $4 k$ for some positive integer $k$. Then there is a nondegenerate symmetric bilinear pairing on the middle cohomology $H^{2 k}(X ; \mathbb{R})$ given by the cup product followed by evaluation on the fundamental class:

$$
\begin{aligned}
H^{2 k}(X ; \mathbb{R}) \otimes H^{2 k}(X ; \mathbb{R}) & \longrightarrow \mathbb{R} \\
\alpha_{1} \otimes \alpha_{2} & \longmapsto\left(\alpha_{1} \smile \alpha_{2}\right)[X] .
\end{aligned}
$$

The signature $\operatorname{Sign}(X)$ of this pairing is called the signature of $X$. (The term "index" is used in place of "signature" in older literature.) Hirzebruch defines the $L$-class as the polynomial in the Pontrjagin classes of $X$ determined by the formal expression

$$
L(X)=\prod_{i=1}^{2 k} \frac{y_{i}}{\tanh y_{i}},
$$

where $y_{i},-y_{i}$ are the Chern roots of the complexified tangent bundle 3 This is analogous to (2.5): one first defines $L(X)$ in case $T X \otimes \mathbb{C} \rightarrow X$ splits as a sum of complex line bundles.

Theorem 2.11 (Hirzebruch's signature theorem). The signature of a closed oriented smooth manifold $X$ is

$$
\operatorname{Sign}(X)=L(X)[X]
$$

Hirzebruch's proof uses Thom's bordism theory [T1 in an essential way. Both sides of (2.12) are invariant under oriented bordism and are multiplicative; for the signature, the former is a theorem of Thom [T2, §IV]. Therefore, it suffices to verify (2.12) on a set of generators of the (rational) oriented bordism ring, which had been computed by Thom. The even projective spaces $\mathbb{C P}^{2 n}$ provide a convenient set of generators, and the proof concludes with the observation that the $L$-class is characterized as evaluating to 1 on these generators. The Todd class enters the proof of Theorem 2.7 in a similar manner-its value on all projective spaces $\mathbb{C P}^{n}$ is 1 and it is characterized by this property.

\footnotetext{
${ }^{3}$ The total Pontrjagin class $p(X)=1+p_{1}(X)+p_{2}(X)+\cdots$ is defined by the expression $\prod\left(1+y_{i}^{2}\right)$.
} 
2.3. Grothendieck's Riemann-Roch theorem. Hirzebruch's Riemann-Roch theorem was extended in a new direction by Grothendieck BS] in 1957. A decisive step was Grothendieck's introduction of $K$-theory in algebraic geometry. Let $X$ be a smooth algebraic variety. Define $K(X)$ as the free abelian group generated by coherent algebraic sheaves on $X$, modulo the equivalence $\mathscr{F} \sim \mathscr{F}^{\prime}+\mathscr{F}^{\prime \prime}$ if there is a short exact sequence $0 \rightarrow \mathscr{F}^{\prime} \rightarrow \mathscr{F} \rightarrow \mathscr{F}^{\prime \prime} \rightarrow 0$. One can replace "coherent algebraic sheaves" by "holomorphic vector bundles" in this definition, and one fundamental result is that the group $K(X)$ is unchanged. Thus Chern classes and the Chern character are defined for elements of $K(X)$. (Grothendieck refines these to take values in the Chow ring of $X$.) If $f: X \rightarrow Y$ is a morphism of varieties, and $\mathscr{F}$ a sheaf over $X$, then $R^{q} f_{*}(\mathscr{F})$ is the sheaf on $Y$ associated to the presheaf $U \mapsto H^{q}\left(f^{-1}(U), \mathscr{F}\right)$. The assignment

$$
f_{!}: \mathscr{F} \longmapsto \sum(-1)^{q} R^{q} f_{*}(\mathscr{F}) \in K(Y)
$$

extends to a homomorphism of abelian groups $f_{!}: K(X) \longrightarrow K(Y)$, as can be seen from the long exact sequence in sheaf cohomology.

Now let $f: X \rightarrow Y$ be a proper morphism between nonsingular irreducible quasiprojective varieties. There is a pushforward $f_{*}$ in cohomology (or on the Chow rings).

Theorem 2.14 (Grothendieck's Riemann-Roch theorem). For $z \in K(X)$ we have

$$
\operatorname{Todd}(Y) \operatorname{ch}\left(f_{!}(z)\right)=f_{*}(\operatorname{Todd}(X) \operatorname{ch}(z)) .
$$

This reduces to Hirzebruch's Riemann-Roch Theorem 2.7 upon taking $Y$ to be a point and $z$ the $K$-theory class of a holomorphic vector bundle.

One route to the Todd class is the special case in which $f: X \rightarrow Y$ is the inclusion of a divisor and $z$ is the class of the structure sheaf $\mathcal{O}_{X}$. Then $R^{q} f_{*}\left(\mathcal{O}_{X}\right)=0$ for $q \geq 1$ and $R^{0} f_{*}\left(\mathcal{O}_{X}\right)$ is $\mathcal{O}_{X}$ extended by zero to $Y$. Let $L \rightarrow Y$ be the line bundle defined by the divisor $X$. Observe that $f^{*}(L) \rightarrow X$ is the normal bundle to $X$ in $Y$. The exact sequence of sheaves

$$
0 \longrightarrow L^{-1} \longrightarrow \mathcal{O}_{Y} \longrightarrow f_{!} \mathcal{O}_{X} \longrightarrow 0
$$

leads to the equality

$$
f_{!} \mathcal{O}_{X}=\mathcal{O}_{Y}-L^{-1}
$$

in $K(Y)$. Set $y=c_{1}(L)$. Then from (2.17),

$$
\operatorname{ch}\left(f_{!}\left(\mathcal{O}_{X}\right)\right)=1-e^{-y} .
$$

On the other hand

$$
f_{*}\left(\operatorname{ch}\left(\mathcal{O}_{X}\right)\right)=f_{*}(1)=y .
$$

Thus $f_{*} \circ \mathrm{ch}=$ chof! up to the Todd class of $L$. To check Theorem 2.14 in this case, rewrite (2.15) using the exact sequence

$$
0 \longrightarrow T X \longrightarrow f^{*} T Y \longrightarrow f^{*} L \longrightarrow 0
$$


of vector bundles on $X$ and the multiplicativity of the Todd genus,

$$
\begin{aligned}
\operatorname{ch}\left(f_{!}(z)\right) & =f_{*}\left(\frac{\operatorname{Todd}(X)}{f^{*} \operatorname{Todd}(Y)} \operatorname{ch}(z)\right) \\
& =f_{*}\left(\frac{1}{f^{*} \operatorname{Todd}(L)} \operatorname{ch}(z)\right) \\
& =\frac{1}{\operatorname{Todd}(L)} f_{*}(\operatorname{ch}(z)) .
\end{aligned}
$$

This is what we checked in (2.18) and (2.19) for $z=\left[\mathcal{O}_{X}\right]$.

It is instructive at this stage to consider the inclusion of the zero section $f: X \rightarrow$ $E$ in a rank $k$ vector bundle $\pi: E \rightarrow X$. Then the sheaf $f_{!} \mathcal{O}_{X}=R^{0} f_{*} \mathcal{O}_{X}$ fits into the exact sequence

$$
0 \longrightarrow \pi^{*} \bigwedge^{k} E^{*} \longrightarrow \pi^{*} \bigwedge^{k-1} E^{*} \longrightarrow \cdots \longrightarrow \pi^{*} E^{*} \longrightarrow \mathcal{O}_{E} \longrightarrow f_{!} \mathcal{O}_{X} \longrightarrow 0
$$

of sheaves over $E$. (Compare (2.16).) Here $E^{*}$ is the (sheaf of sections of the) dual bundle to $\pi: E \rightarrow X$, and the arrows in (2.22) at $e \in E$ are contraction by $e$. Thus in $K(E)$ we have

$$
f_{!}\left(\mathcal{O}_{X}\right)=\bigwedge^{\bullet}\left(E^{*}\right)
$$

where $\bigwedge^{\bullet}\left(E^{*}\right)=\sum(-1)^{k} \bigwedge^{k} E^{*}$ in $K$-theory. Note that $\pi: E \rightarrow X$ is the normal bundle to $X$ in $E$.

2.4. Integrality theorems in topology. One consequence of Hirzebruch's Riemann-Roch Theorem 2.7 is that the characteristic number on the right hand side of (2.8), which a priori is a rational number, is actually an integer. This integer is identified as a sum and difference of dimensions of vector spaces by the left hand side. On the other hand, the right hand side is defined for any almost complex manifold. Hirzebruch was led to ask (as early as 1954) whether the Todd genus $\operatorname{Todd}(X)[X]$ of an almost complex manifold (much less a nonalgebraic complex manifold) is an integer [H3. He also asked analogous questions for real manifolds. Define the $\hat{A}$-class 4 of a real manifold $X^{4 k}$ by the formal expression

$$
\hat{A}(X)=\prod_{i=1}^{2 k} \frac{y_{i} / 2}{\sinh y_{i} / 2},
$$

where $y_{i}$ are the Chern roots. This is a polynomial in the Pontrjagin classes. Then the Todd class of an almost complex manifold can be expressed as

$$
\operatorname{Todd}(X)=e^{c_{1}(X) / 2} \hat{A}(X) .
$$

In particular, $\operatorname{Todd}(X)$ depends only on the Pontrjagin classes and the first Chern class. It is reasonable to speculate that it was (2.25) which motivated Hirzebruch to introduce the $\hat{A}$-class. Furthermore, since the second Stiefel-Whitney class $w_{2}$ is the mod 2 reduction of $c_{1}$, Hirzebruch asked: If a real manifold $X^{4 k}$ has $w_{2}(X)=0$, i.e., if $X$ is a spin manifold, then is $\hat{A}(X)[X]$ an integer.5 This was proved true

\footnotetext{
${ }^{4}$ Hirzebruch had previously defined an $A$-class which differs from the $\hat{A}$-class by a power of 2 , hence the notation $\hat{A}$.

${ }^{5}$ In H3] Hirzebruch only asks a less sharp divisibility question (Problem 7 of that paper). The more precise form came later, along with the more general question: If a closed real manifold $X$ admits an element $c \in H^{2}(X ; \mathbb{Z})$ whose reduction $\bmod 2$ is $w_{2}(X)$, and $\operatorname{Todd}(X)$ is defined by (2.25) (with $c$ replacing $c_{1}(X)$ ), then is $\operatorname{Todd}(X)[X]$ an integer?
} 
(initially up to a power of 2 in [BH2]) by Borel and Hirzebruch [BH3] in the late 1950s using results of Milnor on cobordism [Mi1].

The integrality proved, the obvious question presented itself:

$$
\text { What is the integer } \hat{A}(X)[X] \text { ? }
$$

A first answer to this question came from within algebraic topology, though not from traditional Eilenberg-MacLane cohomology theory. When Atiyah and Hirzebruch learned about Grothendieck's work, they immediately set out to investigate possible ramifications in topology. The first step was to define $K$-theory for arbitrary CW complexes $X$ [AH1]. The definition is as for algebraic varieties, but with "topological vector bundles" replacing "coherent algebraic sheaves". The basic building blocks of topology are the spheres, and the calculation of $K\left(S^{n}\right)$ quickly reduces to that of the stable homotopy groups of the unitary group. By a fortunate coincidence Bott had just computed (in 1957) these homotopy groups B1, B2. His periodicity theorem became the cornerstone of the new topological $K$-theory. What results is a cohomology theory which satisfies all of the Eilenberg-MacLane axioms save one, the dimension axiom. Thus was born "extraordinary cohomology". $K$-theory is the subject of Graeme Segal's paper in this volume Seg4.

Returning to the Grothendieck program, Atiyah and Hirzebruch formulated a version of the Riemann-Roch theorem for smooth manifolds [AH2, [H2]. Let $f: X \rightarrow Y$ be a smooth map between differentiable manifolds, and suppose $f$ is "oriented" in the sense that there exists an element $c \in H^{2}(X ; \mathbb{Z})$ with

$$
c \equiv w_{2}(X)-f^{*} w_{2}(Y) \quad(\bmod 2) .
$$

Recall that Grothendieck's theorem (2.15) is stated in terms of a map $f_{!}: K(X) \rightarrow$ $K(Y)$. In the topological category we cannot push forward vector bundles, as we could sheaves in the algebraic category, so a new construction is needed 6 Here we restrict our attention to embeddings of complex manifolds to simplify the presentation 7 Then (2.17) and (2.23) motivate the definition of $f_{!}$. Let $\pi: E \rightarrow X$ be the normal bundle of $X$ in $Y$. By the tubular neighborhood theorem, we can identify $E$ with a neighborhood $U$ of $X$ in $Y$. The Thom complex $\wedge^{\bullet} E^{*} \rightarrow E$ is defined on the total space of $E$ by contraction (compare (2.22) ):

$$
0 \longrightarrow \pi^{*} \bigwedge^{k} E^{*} \stackrel{\iota(e)}{\longrightarrow} \pi^{*} \bigwedge^{k-1} E^{*} \longrightarrow \cdots \longrightarrow \pi^{*} E^{*} \stackrel{\iota(e)}{\longrightarrow} E \times \mathbb{C} \longrightarrow 0 .
$$

Notice that (2.28) is exact for $e \neq 0$, so the resulting $K$-theory element is supported on $X$. By the tubular neighborhood theorem it is also defined on $U$, and extension by zero yields the desired element $f_{!}(1) \in K(Y)$. If $V \rightarrow X$ is a vector bundle, then $f_{!}(V)$ is defined by tensoring (2.28) with $\pi^{*} V$.

The Atiyah-Hirzebruch Riemann-Roch theorem for smooth manifolds states

$$
\operatorname{ch}\left(f_{!}(z)\right) \operatorname{Todd}(Y)=f_{*}(\operatorname{ch}(z) \operatorname{Todd}(X)), \quad z \in K(X) .
$$

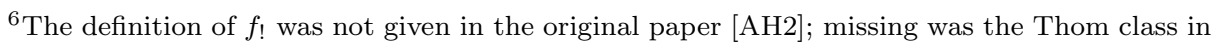
$K$-theory, which is closely related to the symbol of the Dirac operator. The Dirac operator enters the story in the collaboration of Atiyah and Singer (4.1), and then the $K$-theory Thom class and Thom isomorphism appear in ABS §12]. See also the discussion in [Seg4, §1].

${ }^{7}$ General case: Embed a closed manifold $X$ in a sphere, and so factor an arbitrary map $f: X \rightarrow Y$ into an embedding followed by a projection: the composition $X \rightarrow S^{N} \times Y \rightarrow Y$. Bott Periodicity calculates the "shriek map" $K\left(S^{N} \times Y\right) \rightarrow K(Y)$. For embeddings of real manifolds (with an orientation of the normal bundle) Clifford multiplication on spinors replaces (2.28).
} 
Once $f$ ! is defined, the proof is an exercise that compares Thom isomorphisms in $K$-theory and cohomology. Specialize now to $Y=$ pt, and suppose $w_{2}(X)=0$. Choose the orientation class $c \in H^{2}(X ; \mathbb{Z})$ to be zero. Then for $z=0$ in (2.29) we deduce, in view of (2.25), that $\hat{A}(X)[X]=f_{!}(1) \in K(\mathrm{pt}) \cong \mathbb{Z}$ is an integer. This argument by Atiyah and Hirzebruch provided a new proof of the integrality theorem for $\hat{A}$, and also a topological interpretation of the integer $\hat{A}(X)[X]$, so a first answer to (2.26).

Still, that explanation was not considered satisfactory. As reported by Atiyah A1, Hirzebruch realized that the signature is the difference in dimensions of spaces of harmonic differential forms, and he asked for a similar analytic interpretation of the $\hat{A}$-genus $\hat{A}(X)[X]$. Thus when Singer arrived for a sabbatical stay in Oxford in January 1962, the first question Atiyah asked him was, "Why is the $A$-roof genus an integer for a spin manifold?" Singer [S1] responded, "Michael, why are you asking me that question? You know the answer to that." But Atiyah was looking for something deeper, and he immediately had Singer hooked. By March the duo was in possession of the Dirac operator and the index formula. Then, nine months after that initial conversation, Atiyah and Singer completed the first proof of their eponymous index theorem.

\section{AnteCEDEnts in ANALYsis}

The Atiyah-Singer index theorem brings the worlds of algebraic geometry and algebraic topology together with the worlds of differential geometry and global analysis. Our introduction to the latter in $\$ 3.1$ begins with foundational theorems about harmonic differential forms and their relationship to cohomology. Geometric elliptic differential operators on Riemannian manifolds play a central role. We take up more general elliptic operators in $\$ 3.2$, where we also recall basic facts about Fredholm operators. The Fredholm index, an integer-valued deformation invariant of a Fredholm operator, is the eponymous character of index theory. In 3.3 we give the reader an inkling of the activity around indices of elliptic operators during the years $1920-1963$.

3.1. De Rham, Hodge, and Dolbeault. We begin with the de Rham and Hodge theorems, which exemplify the relationship between elliptic linear differential equations and topology. Let $X$ be a smooth $n$-dimensional manifold, and consider the complex of differential forms

$$
\Omega^{0}(X) \stackrel{d}{\longrightarrow} \Omega^{1}(X) \stackrel{d}{\longrightarrow} \cdots \stackrel{d}{\longrightarrow} \Omega^{n}(X)
$$

where $d$ is the exterior derivative of Elie Cartan. The de Rham cohomology vector spaces are defined as the quotients

$$
H_{\mathrm{DR}}^{q}(X)=\frac{\operatorname{ker}\left[d: \Omega^{q}(X) \rightarrow \Omega^{q+1}(X)\right]}{\text { image }\left[d: \Omega^{q-1}(X) \rightarrow \Omega^{q}(X)\right]}, \quad 0 \leq q \leq n .
$$

The theorem de Rham proved in his 1931 thesis deR states that for each $q$ there is a natural isomorphism $H_{\mathrm{DR}}^{q}(X) \stackrel{\cong}{\longrightarrow} H^{q}(X ; \mathbb{R})$ of the de Rham cohomology with real cohomology defined via singular cochains. (This is modern language; de Rham proved that there is a closed form with specified periods, unique modulo exact forms.) Notice that $H_{\mathrm{DR}}^{q}(X)$ is defined using a differential operator, whereas $H^{q}(X ; \mathbb{R})$ comes from topology. Hodge, motivated by questions in algebraic geometry, proved that on a closed Riemannian manifold there is a unique "best" 
form in each cohomology class. Namely, on an oriented Riemannian manifold $X$ Hodge defined a duality operation $*: \Omega^{q}(X) \rightarrow \Omega^{n-q}(X)$, and for closed manifolds he argued [Hod] that in each de Rham cohomology class there is a unique form $\omega$ satisfying

$$
d \omega=0, \quad d(* \omega)=0 .
$$

These harmonic differential forms are solutions to the elliptic Hodge-Laplace equation

$$
\Delta \omega=\left(d d^{*}+d^{*} d\right) \omega=0,
$$

which on a closed manifold is equivalent to the pair of equations (3.3). The number of linearly independent solutions - the dimension of the vector space $\mathscr{H}^{q}(X)$ of solutions - equals a topological invariant, the Betti number $b_{q}=\operatorname{dim} H^{q}(X ; \mathbb{R})$. There is a stronger statement, namely an isomorphism $\mathscr{H}^{q}(X) \stackrel{\cong}{\longrightarrow} H^{q}(X ; \mathbb{R})$. Neither statement generalizes to arbitrary elliptic differential operators; rather, the index theorem in this situation computes the alternating sum of dimensions of spaces of harmonic forms, a familiar topological invariant,

$$
\sum_{q=0}^{n}(-1)^{q} \operatorname{dim} \mathscr{H}^{q}(X)=\operatorname{Euler}(X),
$$

where $\operatorname{Euler}(X)$ is the Euler number of $X$. (Compare (2.3).)

We can express the left hand side of (3.5) as the index of an elliptic operator, namely

$$
d+d^{*}: \Omega^{\text {even }}(X) \longrightarrow \Omega^{\text {odd }}(X) .
$$

Its formal adjoint is $d+d^{*}: \Omega^{\text {odd }}(X) \rightarrow \Omega^{\text {even }}(X)$, and we identify the cokernel of (3.6) with the kernel of the adjoint. If $\operatorname{dim} X$ is even, then a different $\mathbb{Z} / 2 \mathbb{Z}$ grading 8 on complex differential forms $\Omega \bullet(X, \mathbb{C})$ leads to another elliptic operator [AS3, §6],

$$
d+d^{*}: \Omega^{+}(X, \mathbb{C}) \longrightarrow \Omega^{-}(X, \mathbb{C}),
$$

the signature operator, whose index on a closed manifold of dimension divisible by 4 is the signature of the pairing (2.9).

Let $X$ be a closed $n$-dimensional complex manifold, and let $V \rightarrow X$ be a holomorphic vector bundle. Then the sheaf cohomology groups $H^{q}(X, V)$ used in $\$ 2.2$ are isomorphic to the cohomology groups of the $\vec{d}$-complex

$$
\Omega^{0,0}(X, V) \stackrel{\bar{d}}{\longrightarrow} \Omega^{0,1}(X, V) \stackrel{\bar{d}}{\longrightarrow} \cdots \stackrel{\bar{d}}{\longrightarrow} \Omega^{0, n}(X, V),
$$

as was proved by Dolbeault. If $\operatorname{dim} X=1$, then (3.8) reduces to a single elliptic operator, and for $V \rightarrow X$ the line bundle associated to a divisor $D$ on $X$ the vector space $\mathcal{L}(D)$ of $\$ 2.1$ is naturally isomorphic to the kernel of $d$. If $X$ is Kähler, then Hodge theory implies that the Dolbeault cohomology vector spaces are isomorphic to vector spaces of complex harmonic differential forms. Putting these theorems together, we deduce that on a Kähler manifold the holomorphic

\footnotetext{
${ }^{8}$ The grading $\Omega^{\bullet}(X, \mathbb{C})=\Omega^{+}(X, \mathbb{C}) \oplus \Omega^{-}(X, \mathbb{C})$ is the eigenspace decomposition of the involution $\tau$ on $\Omega^{\bullet}(X, \mathbb{C})$ defined by $\tau(\omega)=i^{p(p-1)+\ell} * \omega$, where $\operatorname{dim} X=2 \ell$.
} 
Euler characteristic (2.3) is the alternating sum of dimensions of spaces of harmonic forms. Hirzebruch's Riemann-Roch Theorem 2.7 is a topological formula for this analytic quantity:

$$
\sum_{q=0}^{n} \operatorname{dim} \mathscr{H}^{0, q}(X)=\chi(X, V)=\operatorname{Todd}(X) \operatorname{ch}(V)[X] .
$$

As mentioned at the end of §2, Hirzebruch's signature theorem can also be interpreted in terms of harmonic differential forms.

3.2. Elliptic differential operators and the Fredholm index. We set up the index problem on a closed $n$-manifold $X$. Let $E^{0}, E^{1} \rightarrow X$ be vector bundles over $X$, and suppose $P: C^{\infty}\left(X, E^{0}\right) \rightarrow C^{\infty}\left(X, E^{1}\right)$ is a linear differential operator of order $m$. In local coordinates $x^{1}, \ldots, x^{n}$ on $X$, for $u \in C^{\infty}\left(X, E^{0}\right)$ a smooth section of $E^{0} \rightarrow X$ we have

$$
P u=a^{i_{1} i_{2} \cdots i_{m}} \frac{\partial^{m} u}{\partial x^{i_{1}} \partial x^{i_{2}} \cdots \partial x^{i_{m}}}+\text { lower-order terms, }
$$

where $a^{i_{1} i_{2} \cdots i_{m}}$ is a bundle map $E^{0} \rightarrow E^{1}$ depending symmetrically on the $i_{j}$, and we sum over the indices $1 \leq i_{j} \leq n$. This highest-order term transforms as a tensor under coordinate changes, so it defines a global bundle map

$$
\sigma(P): \operatorname{Sym}^{m}\left(T^{*} X\right) \otimes E^{0} \longrightarrow E^{1},
$$

called the symbol of $P$. View $\sigma(P)$ as a homogeneous polynomial of degree $m$ in $T^{*} X$ with values in $\operatorname{Hom}\left(E^{0}, E^{1}\right)$. The differential operator $P$ is elliptic if its symbol is invertible; that is, if for each $x \in X$ and nonzero $\theta \in T_{x}^{*} X$, the linear map $\sigma(P)(\theta, \ldots, \theta): E_{x}^{0} \rightarrow E_{x}^{1}$ is invertible. It follows from elliptic theory that $P$ has finite-dimensional kernel and cokernel. (This relies on the compactness of $X$.) The (Fredholm) index of $P$ is

$$
\text { ind } P=\operatorname{dim} \operatorname{ker} P-\operatorname{dim} \text { coker } P \text {. }
$$

Elliptic theory proves that the extension of $P$ to appropriate Sobolev spaces is a Fredholm operator. Recall that a Fredholm operator $H^{0} \rightarrow H^{1}$ is a bounded linear operator between Hilbert spaces which has closed range, finite-dimensional kernel, and finite-dimensional cokernel. (The definition generalizes to Banach spaces and beyond.) The index of a Fredholm operator is defined9 by (3.12). The space $\operatorname{Hom}\left(H^{0}, H^{1}\right)$ of continuous linear maps has a Banach space structure defined by the operator norm, and the open subspace $\operatorname{Fred}\left(H^{0}, H^{1}\right) \subset \operatorname{Hom}\left(H^{0}, H^{1}\right)$ of Fredholm operators has nontrivial homotopy groups of unbounded degree. In particular, the index

$$
\text { ind: } \pi_{0} \operatorname{Fred}\left(H^{0}, H^{1}\right) \longrightarrow \mathbb{Z}
$$

is an isomorphism. In other words, the numerical index is a complete deformation invariant of a single Fredholm operator. Furthermore, the action of compact operators by translation preserves the subspace of Fredholm operators, hence the index is invariant under this translation.

\footnotetext{
${ }^{9}$ A linear map $T: V^{0} \rightarrow V^{1}$ between finite-dimensional vector spaces is an element of $\left(V^{1}\right) \otimes$ $\left(V^{0}\right)^{*}$, so it stands to reason that the sign in (3.12) should have been the opposite: the dual-or minus - is the domain, not the codomain. The usual sign convention causes headaches down the road, for example in the theory of determinants. On the other hand, one could argue for the usual sign convention by rewriting a single operator as a 2-term complex in degrees 0 and 1 , and then the usual signs for the index (as in (2.3) ) reduce to (3.12).
} 
Elliptic theory implies that the lower-order terms of an elliptic differential operator (3.10) on a smooth manifold are compact relative to the highest-order term, which is essentially the symbol (3.11). It follows that the index of an elliptic differential operator is an invariant of its symbol. Furthermore, a continuous path of elliptic differential operators induces a continuous path of Fredholm operators and of symbols (in suitable topologies). The index is unchanged under such deformations and, in fact, only depends on the homotopy class of the symbol. The Atiyah-Singer index theorem provides a formula for the index in terms of the homotopy class of the symbol.

3.3. Index problems for elliptic operators. There is a long and rich history of index theorems for linear elliptic problems in the first half of the $20^{\text {th }}$ century. Many are subsumed by the Atiyah-Singer index theorem and its extension to manifolds with boundary (\$5.2). The articles by Agranovich Agr and Seeley [Se1] are excellent guides to this history. The first index theorem is contained in a 1920 paper of Fritz Noether [N]. (This is credited in modern references such as [E, AM].) Moreover, this paper seems to be the origin of the Fredholm index. In fact, in the older literature the following terminology is sometimes used: a linear operator is said to obey the "Noether property" if it is Fredholm, in which case "Fredholm" is reserved for an operator of index zero (which then satisfies the "Fredholm alternative").

One case of Noether's work is an index formula for Toeplitz operators. Let $S^{1} \subset \mathbb{C}$ be the unit circle, and let $f: S^{1} \rightarrow \mathbb{C}^{\times}$be a smooth nonzero complexvalued function. The Toeplitz operator $T_{f}: \mathscr{H} \rightarrow \mathscr{H}$ is defined on the Hilbert space $\mathscr{H}$ of $L^{2}$ holomorphic functions on the closure $\bar{\Omega}$ of the unit disk $\Omega \subset \mathbb{C}$. By Fourier series $\mathscr{H}$ sits as a subspace in $\widetilde{\mathscr{H}}=L^{2}\left(S^{1}, \mathbb{C}\right)$. Let $i: \mathscr{H} \hookrightarrow \widetilde{\mathscr{H}}$ be the inclusion, and let $\pi: \widetilde{\mathscr{H}} \rightarrow \mathscr{H}$ be the orthogonal projection. Then $T_{f}=\pi \circ M_{f} \circ i$ is the compression of the multiplication operator $M_{f}: \widetilde{\mathscr{H}} \rightarrow \widetilde{\mathscr{H}}$ to $\mathscr{H}$.

Theorem 3.14 (Noether, 1920). $T_{f}$ is Fredholm with index minus the winding number of $f$.

The reader may wish to compute the index explicitly for $f_{n}(z)=z^{n}, n \in \mathbb{Z}$. Theorem 3.14 was rediscovered by Gohberg and Krein [GK], and there are index theorems for Toeplitz operators in arbitrary dimensions, for example in Boutet de Monvel [BdM].

In 1960, Gelfand [G] observed that the index is a homotopy invariant, and he posed the general problem of computing a topological formula for the index. It seems that Atiyah and Singer were unaware of these events in Russia when they embarked on the journey which led to the index theorem, though they became aware of them during a visit by Smale to Oxford [A1. Gelfand's paper, and some of its antecedents which solve special cases of the index problem, are cited at the beginning of the Atiyah-Singer announcement of their general index theorem AS1].

\section{THE INDEX THEOREM AND PROOFS}

We arrive at the Atiyah-Singer index theorem for a single elliptic operator. It was announced in AS1] in 1963. Atiyah and Singer's first proof (\$4.2), modeled on Hirzebruch's cobordism proofs of his signature and Riemann-Roch theorems, was not written up by them but rather was published in Palais $\mathrm{Pa}$ as a series of pieces by many contributors in a volume which remains a valuable reference. The second 
proof (\$4.5), modeled more on Grothendieck, appeared in 1968 in the first AS2], a series of papers by Atiyah and Singer. Subsequent papers treat variations and generalizations. We begin in $\$ 4.1$ with the Dirac operator in Riemannian geometry. It is the analogue of Dirac's operator in Lorentz geometry, and it is central in many contexts in geometry and physics as well as in general index theory. In a different direction, pseudodifferential operators play an important role in both proofs of the index theorem; we give a brief introduction in $\$ 4.3$. In $\$ 4.4$ we list a few early applications of the index theorem.

4.1. The Dirac operator. In 1928 Dirac D] introduced his equation as part of his relativistic theory of electrons. Dirac worked on Minkowski spacetime. The analogue of Dirac's line of inquiry for Euclidean space $\mathbb{E}^{n}$ with standard coordinates $x^{1}, \ldots, x^{n}$ asks for a first-order differential operator

$$
D=\gamma^{1} \frac{\partial}{\partial x^{1}}+\cdots+\gamma^{n} \frac{\partial}{\partial x^{n}}
$$

whose square is the Laplace operator

$$
\Delta=-\left\{\left(\frac{\partial}{\partial x^{1}}\right)^{2}+\cdots+\left(\frac{\partial}{\partial x^{n}}\right)^{2}\right\} .
$$

(In Minkowski spacetime the elliptic Laplace operator (4.2) is replaced by the hyperbolic wave operator.) Assume that $\gamma^{1}, \ldots, \gamma^{n}$ are constant functions on $\mathbb{E}^{n}$. Then the differential equation

$$
D^{2}=\Delta
$$

is equivalent to the system of algebraic equations

$$
\gamma^{i} \gamma^{j}+\gamma^{j} \gamma^{i}=-2 \delta^{i j}=\left\{\begin{array}{rl}
-2, & i=j ; \\
0, & i \neq j,
\end{array} \quad 1 \leq i, j \leq n .\right.
$$

There are no scalar solutions if $n \geq 2$, but there are matrix solutions. Let 10 Cliff $_{-n}$, the Clifford algebra $[\mathrm{Cl}, \mathrm{Ca}, \mathrm{BW}, \overline{\mathrm{Ch}}, \overline{\mathrm{ABS}}$, be the unital algebra over $\mathbb{R}$ generated by $\gamma^{1}, \ldots, \gamma^{n}$ subject to the relations (4.4). A matrix solution to (4.4) defines a Cliff $_{-n}$-module. The spin group $\operatorname{Spin}_{n}$ is a double cover of the special orthogonal group $\mathrm{SO}_{n}$, and $\mathrm{Spin}_{n}$ is a subgroup of the units in Cliff $-n$, much as $\mathrm{SO}_{n}$ is a subgroup of the units in the algebra of $n \times n$ real matrices. Clifford modules restrict to special representations of $\operatorname{Spin}_{n}$ called spin or spinor representations.

Why does the spin group enter the quest to identify the integer $\hat{A}(X)[X]$ ? One key is the formula (2.24) for the $\hat{A}$-genus. Assuming $n$ is even, the group $\operatorname{Spin}_{n}$ has two distinguished inequivalent irreducible complex representations $\mathbb{S}^{0}, \mathbb{S}^{1}$, and the difference of the characters of $\mathbb{S}^{0}$ and $\mathbb{S}^{1}$ is an upside-down variant of the $\hat{A}$ genus (2.24), namely

$$
\prod_{i=1}^{n / 2} \frac{\sinh y_{i} / 2}{1 / 2}
$$

where now $y_{1}, \ldots, y_{n}$ are a basis of characters of a maximal torus of $\operatorname{Spin}_{n}$. Second, the spin condition was known to be related to the integrality of the $\hat{A}$-genus, as explained in 2.4. Perhaps these considerations led Atiyah and Singer to construct

\footnotetext{
${ }^{10}$ To define the closely related Clifford algebra Cliff $+n$, change the - sign in relation (4.4) to $\mathrm{a}+$ sign. Both Clifford algebras appear in 5.4
} 
the Dirac operator on a Riemannian spin manifold. Observe too that on a Riemannian manifold the square of the first-order differential operator $d+d^{*}$ is the Hodge-Laplace operator (3.4), so in that sense $d+d^{*}$ is already a Dirac operator. On a Kähler manifold, the same holds for $d+d^{*}$.

The Dirac operator on an even-dimensional Riemannian spin manifold $X^{n}$ is a main character in the index theorem, so we give the definition here. Let $\mathrm{SO}(X) \rightarrow$ $X$ be the principal $\mathrm{SO}_{n}$-bundle whose fiber at $x \in X$ consists of oriented isometries $\mathbb{R}^{n} \rightarrow T_{x} X$. The spin structure is a double cover $\operatorname{Spin}(X) \rightarrow \mathrm{SO}(X)$ together with a compatible principal $\operatorname{Spin}_{n}$-bundle structure on the composition $\operatorname{Spin}(X) \rightarrow$ $\mathrm{SO}(X) \rightarrow X$. Use the complex spin representations $\mathbb{S}^{0}, \mathbb{S}^{1}$ to construct associated complex vector bundles $\mathbb{S}_{X}^{0}, \mathbb{S}_{X}^{1} \rightarrow X$; sections of these vector bundles are called spinors or spinor fields. The Levi-Civita connection on $X$ induces a covariant derivative $\nabla$ on each spinor bundle. The Dirac operator is

$$
D_{X}=c \circ \nabla: C^{\infty}\left(X, \mathbb{S}_{X}^{0}\right) \longrightarrow C^{\infty}\left(X, \mathbb{S}_{X}^{1}\right),
$$

where $c$ is Clifford multiplication, induced from a $\operatorname{Spin}_{n}$-equivariant linear map $\mathbb{R}^{n} \otimes \mathbb{S}^{0} \rightarrow \mathbb{S}^{1}$. There is also a Clifford multiplication $\mathbb{R}^{n} \otimes \mathbb{S}^{1} \rightarrow \mathbb{S}^{0}$, and both together give $\mathbb{S}^{0} \oplus \mathbb{S}^{1}$ the structure of a Cliff $n_{n}$-module. This construction is not quite canonical, since the irreducible representations $\mathbb{S}^{0}, \mathbb{S}^{1}$ are only determined uniquely up to tensoring by a line. In $\$ 5.4$ we introduce the Clifford linear Dirac operator, which is canonical.

The de Rham (3.1) and Dolbeault (3.8) operators have similar descriptions, and so (3.5) and (3.9) motivate a conjectural index formula for the Dirac operator. Let $\mathscr{H}^{ \pm}(X)$ be the complex vector spaces of harmonic spinors, i.e., solutions $\psi$ to $D \psi=0$. The conjectured index formula is

$$
\operatorname{dim} \mathscr{H}^{+}(X)-\operatorname{dim} \mathscr{H}^{-}(X)=\hat{A}(X)[X] .
$$

For an investigation into various aspects of harmonic spinors, see the DPhil thesis [Hi] of Atiyah's student Nigel Hitchin.

4.2. First proof: cobordism. Let $X$ be a closed oriented manifold; let $E^{0}, E^{1} \rightarrow$ $X$ be complex vector bundles; and let $P: C^{\infty}\left(X, E^{0}\right) \rightarrow C^{\infty}\left(X, E^{1}\right)$ be an elliptic differential operator. The analytic index of $P$ is the Fredholm index defined in (3.12). The topological index - in cohomological form - is the following. Ellipticity implies that the symbol $\sigma(P)$ in (3.11) restricted to the nonzero vectors $T^{*} X \backslash 0$ is an isomorphism. Therefore, $\sigma(P)$ defines a relative $K$-theory class in $K^{0}\left(T^{*} X, T^{*} X \backslash 0\right)$. The Chern character maps $K$-theory to rational cohomology, and then the inverse of the Thom isomorphism

$$
\phi: H^{\bullet}(X ; \mathbb{Q}) \stackrel{\cong}{\longrightarrow} H^{\bullet+\operatorname{dim} X}\left(T^{*} X, T^{*} X \backslash 0 ; \mathbb{Q}\right)
$$

brings us to the cohomology of the base $X$. The topological index is the cup product of the Todd class of the complexified tangent bundle with the image of $\sigma(P)$ under the Chern character and Thom isomorphism. The index theorem asserts that the analytic and topological indices are equal.

Theorem 4.9 (Atiyah-Singer index theorem AS1]). The index of $P$ is

$$
\text { ind } P=\left(\operatorname{Todd}(T X \otimes \mathbb{C}) \smile \phi^{-1} \operatorname{ch} \sigma(P)\right)[X] .
$$

In the remainder of this section we sketch the main ideas which enter the proof of Theorem 4.9 . 
Remark 4.11. The $K$-theoretic formula for the index (\$4.5) is more natural and lends itself to many generalizations. This fits with Atiyah's philosophy that $K$ theory, based on linear algebra, is more elementary than cohomology. Certainly it is the form of algebraic topology which most closely matches linear differential operators.

Now to the proof. At the end of $\$ 3.2$ we indicated that the analytic index depends only on the homotopy class of the symbol $\sigma(P)$. Atiyah and Singer introduce the abelian group

$$
\operatorname{Ell}(X)=K^{0}\left(T^{*} X, T^{*} X \backslash 0\right)
$$

of elliptic symbol classes. It is a module over the ring $K^{0}(X)$, by tensor product, and the cyclic module $K^{0}(X) \sigma_{0}$ generated by the symbol class of the signature operator is a subgroup of finite index. This reduces the problem on a fixed manifold $X$ to the signature operator twisted by a vector bundle $W \rightarrow X$. However, to carry through this argument, one needs that every element of $\operatorname{Ell}(X)$ is the symbol of an elliptic operator, and furthermore if the symbols of elliptic operators $P_{0}, P_{1}$ define the same element of $\operatorname{Ell}(X)$, then there is a homotopy $P_{0} \sim P_{1}$. Differential operators are too rigid for these properties to hold, and a critical move in the proof is the introduction of pseudodifferential operators, which we discuss briefly in the next section.

Remark 4.13. If $X$ is a spin manifold, then $\operatorname{Ell}(X)$ is the cyclic $K^{0}(X)$-module generated by the symbol of the Dirac operator. This fact is an expression of Bott periodicity, as realized in $K$-theory.

Bordism enters the proof at this stage. For the signature operator twisted by a vector bundle $W \rightarrow X$, both sides of (4.10) are viewed as functions of a pair $(X, W)$, where we only use the equivalence class $[W] \in K^{0}(X)$. A crucial step is the proof that each side is a bordism invariant of $(X, W)$. This is straightforward for the cohomological formula on the right hand side. For the analytic index, suppose $(X, W)$ is the boundary of a pair $(Y, U)$, where $Y$ is a compact oriented manifold and $U \rightarrow Y$ a complex vector bundle. Atiyah and Singer introduce an elliptic differential operator $D_{U}$ on $Y$ whose boundary operator on $X$ is the twisted signature operator. They specify a local elliptic boundary condition, and prove

(i) $\operatorname{ind}_{Y} D_{U}=\operatorname{ind}_{X} D_{W}$, and

(ii) $\operatorname{ind}_{Y} D_{U}=0$.

With bordism invariance in hand, it remains to compute a basis for the rational vector space of bordism classes of pairs $(X, W)$ and verify (4.10) for those.

The journey from this rough sketch to a complete proof is replete with interesting detours in analysis, geometry, and topology.

4.3. Pseudodifferential operators. Let $P$ be a differential operator of order $m$ on $\mathbb{R}^{n}$, as in (3.10). Its action on a smooth function $u$ of compact support is conveniently written in terms of the Fourier transform $\hat{u}$ :

$$
P u(x)=(2 \pi)^{-n} \int_{\mathbb{R}^{n *}} p(x, \xi) \hat{u}(\xi) e^{i\langle x, \xi\rangle} d \xi, \quad x \in \mathbb{R}^{n},
$$

where for each $x \in \mathbb{R}^{n}$ the function $p(x, \xi)$ is a polynomial of degree $m$ in $\xi \in \mathbb{R}^{n *}$. A generalization, going back to Mikhlin [Mik and to Calderón and Zygmund [CZ], allows more general (total) symbols $p$, with uniform bounds on the behavior of $p$ and its derivatives as $|\xi| \rightarrow \infty$. One motivation comes from elliptic operators: a 
parametrix - an approximate inverse - is a linear operator of this form. Also, these operators have a Schwartz kernel which is smooth away from the diagonal, and this leads to good properties. (The Schwartz kernel of a differential operator is zero off the diagonal.) The theory of pseudodifferential operators, and their globalization to smooth manifolds, is treated in papers of Seeley [Se2, Se3, Hörmander [Ho2, Ho3, Kohn and Nirenberg [KN], and Palais and Seeley [PS], among others. This is only a very small sample of the extensive literature.

If $P$ is a differential operator of order $m$, so $p(x, \xi)$ is a degree $m$ polynomial in $\xi$ for each $x$, then the principal or top-order symbol of $P$ is the homogeneous polynomial of degree $m$ given as

$$
\sigma(P)(x, \xi)=\lim _{\lambda \rightarrow \infty} \frac{p(x, \lambda \xi)}{\lambda^{m}} .
$$

Restrict to pseudodifferential operators with symbol $p$ for which the limit (4.15) exists. Then the principal symbol is defined, in global form (3.11) on a smooth manifold, and ellipticity is as before invertibility of the principal symbol. The surjectivity and continuity of the principal symbol map on elliptic pseudodifferential operators are crucial ingredients in the proof of the index theorem.

We remark that in the Atiyah-Bott [AB1 and Atiyah-Singer AS2, AS4 papers on index theory, the global theory of pseudodifferential operators is expanded further.

4.4. A few applications. One immediate consequence of the Atiyah-Singer Index Theorem 4.9, is the index formula for the Dirac operator (4.7). This provides an analytic interpretation of the $\hat{A}$-genus of a spin manifold, hence an answer to (2.26).

Several additional consequences are described in AS1. As already mentioned, Hirzebruch's signature theorem 2.11 is a special case. So is his Riemann-Roch Theorem 2.7 but the more flexible techniques of Atiyah and Singer prove it for arbitrary closed complex manifolds, a powerful generalization from projective algebraic manifolds. Finally, for systems of elliptic operators on $X$-for $E^{0}, E^{1} \rightarrow X$ trivial vector bundles - the index vanishes given appropriate inequalities between $\operatorname{dim} X$ and $\operatorname{rank} E^{0}=\operatorname{rank} E^{1}$, a result which connects Theorem 4.9 to the PDE literature of the period. In particular, the index vanishes for an elliptic operator acting on a single function.

As new index theorems proliferate, so too do applications, as we will see in $\$ 5$,

4.5. Second proof: $K$-theory. Recall the pushforward (2.13) which occurs in Grothendieck's version of the Riemann-Roch theorem. Let $X$ be a compact projective variety. For the special case of the unique map $f: X \rightarrow \mathrm{pt}$, the pushforward $f_{!}: K(X) \rightarrow K(\mathrm{pt}) \cong \mathbb{Z}$ computes the Euler characteristic (2.3) of a holomorphic vector bundle $V \rightarrow X$. According to the Dolbeault theorem, the sheaf cohomology groups are isomorphic to the cohomology groups of the $d$-complex (3.8). For smooth manifolds and smooth maps, Atiyah and Hirzebruch found a topological pushforward in one of their first works on $K$-theory, and it is designed to match Grothendieck's $f_{!}$in this situation. (See the text surrounding (2.27).) There is also an analytic interpretation. Namely, on a Kähler manifold $X$ the Dolbeault cohomology group $H_{\bar{d}}^{0, q}(X, V)$ is isomorphic to the kernel of the elliptic operator $d+d^{*}$ on $(0, q)$-forms, hence the Euler characteristic (2.3) is the index of

$$
d+d^{*}: \Omega^{0, \text { even }}(X, V) \longrightarrow \Omega^{0, \text { odd }}(X, V) .
$$


That index is an analytic pushforward of $V \rightarrow X$ under the map $f$. The RiemannRoch-de Rham-Hodge-Dolbeault-Hirzebruch theorems imply the equality of analytic and topological pushforwards.

The $K$-theory form of the Atiyah-Singer index theorem is a generalization for arbitrary elliptic (pseudo)differential operators. Recall from $\$ 4.2$ that the "homotopy class" of the symbol $\sigma(P)$ of an elliptic differential operator $P$ on a smooth manifold $X$ is an element $[\sigma(P)] \in K^{0}\left(T^{*} X, T^{*} X \backslash 0\right)$. Atiyah and Singer define two homomorphisms

$$
\text { ind : } K^{0}\left(T^{*} X, T^{*} X \backslash 0\right) \longrightarrow \mathbb{Z} \text {. }
$$

The analytic index a-ind takes a symbol class $\sigma$ to the index ind $P$ of any elliptic pseudodifferential operator $P$ with $[\sigma(P)]=\sigma$. The topological index t-ind is similar to the topological pushforward. It is based on the Thom isomorphism in $K$-theory, which in turn rests on Bott periodicity.

Theorem 4.18 (Atiyah and Singer 1967). a-ind = t-ind.

This is equivalent to the cohomological Theorem 4.9. but as we will see in \$5 the naturalness of the $K$-theory formulation and proof lend themselves to many generalizations.

Theorem 4.18 is the subject of AS2]. There is a concise summary of the proof idea in $\S 1$ of that paper, though the actual proof follows a slightly different arc. In essence, Atiyah and Singer uniquely characterize "index homomorphisms" (4.17) by a short list of axioms, which they verify that a-ind and t-ind satisfy. Beyond normalization axioms, two main properties of the index feature in the proof. An excision axiom extends the index to compactly supported symbols on arbitrary (potentially noncompact) manifolds $U$, at least if $U$ is embeddable in a compact manifold, by asserting the independence of the index of the extension by zero of a compactly supported symbol on $U$ to a symbol on an ambient compact manifold. A multiplicative axiom tells a product formula for (twisted) product symbols. A robust global theory of pseudodifferential operators is used at this point in the proof.

\section{VARIATIONS ON THE THEME}

After the initial Atiyah-Singer work, index theory branched out in multiple directions. Atiyah was at the center of many of these developments, which we can only touch upon in this section. We begin in 5.1 with Atiyah and Bott's generalization of the classical Lefschetz fixed point theorem to elliptic complexes. Its manifold applications include a geometric proof of Weyl's character formula in the theory of compact Lie groups. The link between the Atiyah-Singer $K$-theoretic framework for index theory and the index theory for elliptic operators which preceded it is the extension of the Atiyah-Singer theorem to compact manifolds with boundary, carried out jointly with Bott as we recount in \$5.2. The basic Atiyah-Singer theorem applies to complex elliptic operators. There is an important refinement (\$5.3) to real elliptic operators which, naturally, links to the real version of $K$-theory introduced by Atiyah at this time. There is a canonical real Dirac operator on a Riemannian spin manifold - perhaps not as oft-used as it deserves to be - so we take the opportunity in $\$ 5.4$ to expose it. Grothendieck's dictum to do geometry over a base, not just over a point, leads to an index theorem for families of elliptic operators. As Grothendieck's philosophy promises, this theorem, described in 55.5 , 
appears often in geometry and physics. Finally, in $\$ 5.6$ we introduce an index theorem of Atiyah's which launched an entirely new branch of the subject, though not one he himself pursued.

5.1. Equivariant index theorems. Recall the classical Lefschetz fixed point theorem. Let $X$ be a smooth compact manifold, and let $f: X \rightarrow X$ be a diffeomorphism with isolated fixed points. The subset $\operatorname{Fix}(f) \subset X$ is then finite, and our aim might be to compute its cardinality. But the cardinality is not a deformation invariant - under deformation, pairs of fixed points undergo birth and death - hence there is not a topological formula. However, if we count with signs, weighting each $x \in \operatorname{Fix}(f)$ by 11

$$
\epsilon_{x}=\operatorname{sign} \operatorname{det}\left(1-d f_{x}\right) \in\{-1,+1\},
$$

then the Lefschetz fixed point theorem asserts

$$
\sum_{x \in \operatorname{Fix}(f)} \epsilon_{X}=\sum_{q=0}^{\operatorname{dim} X}(-1)^{q} \operatorname{Tr}\left(\left.f^{*}\right|_{H^{q}(X ; \mathbb{R})}\right),
$$

where the right hand side is the alternating sum of traces of $f$ acting on the $q$ th real cohomology.

In 1965 Atiyah and Bott formulated and proved a generalization to elliptic operators and elliptic complexes. Let $X$ be a closed manifold; let $E^{0}, E^{1} \rightarrow X$ be vector bundles; let $f: X \rightarrow X$ be a diffeomorphism with isolated fixed points; let $f^{i}: E^{i} \rightarrow E^{i}, i=0,1$, be lifts of $f$; and let $P: C^{\infty}\left(X, E^{0}\right) \rightarrow C^{\infty}\left(X, E^{1}\right)$ be an elliptic differential operator which commutes with $f^{i}$. Define

$$
\nu_{x}=\frac{\operatorname{Tr}\left(\left.f^{0}\right|_{E_{x}^{0}}\right)-\operatorname{Tr}\left(\left.f^{1}\right|_{E_{x}^{1}}\right)}{\left|\operatorname{det}\left(1-d f_{x}\right)\right|}, \quad x \in \operatorname{Fix}(f) .
$$

Theorem 5.4 (Atiyah and Bott [AB1]). We have

$$
\sum_{x \in \operatorname{Fix}(f)} \nu_{x}=\operatorname{Tr}\left(\left.f^{0}\right|_{\text {ker } P}\right)-\operatorname{Tr}\left(\left.f^{1}\right|_{\text {coker } P}\right) .
$$

We will not comment on the proof here, which also involves pseudodifferential operators, but instead tell some applications.

In their proof of the index theorem modeled after Grothendieck (\$4.4), Atiyah and Singer AS2 incorporate the action of a compact Lie group $G$ on an elliptic operator $P$ on a compact manifold $X$. The Atiyah-Bott setup overlaps in case their diffeomorphism $f$ of $X$ generates a (compact) torus of diffeomorphisms. In the Atiyah-Singer case, since $G$ commutes with $P$, it acts on ker $P$ and coker $P$, so the formal difference - the index - is a virtual representation of $G$. Hence it defines a class in the representation $\operatorname{ring} R(G)$. On the other hand, the symbol $\sigma(P)$ also commutes with $G$, and so it defines a class in equivariant $K$-theory, an important extension of topological $K$-theory which was investigated by Atiyah's student Graeme Segal in his DPhil thesis Seg1. The topological index t-ind, executed in equivariant $K$-theory, also produces an element of $R(G)$. The equivariant extension of Theorem 4.18 identifies these elements of $R(G)$. Atiyah and Segal ASeg apply the localization theorem in equivariant $K$-theory [Seg1] to derive a Lefschetz-type

\footnotetext{
${ }^{11}$ under the assumption that $d f_{x}$ has no nonzero fixed vectors for all $x \in \operatorname{Fix}(f)$.
} 
formula, and in particular they recover Theorem 5.4 in case $f$ generates a compact Lie group (torus) of diffeomorphisms.

These various Lefschetz formulæ have many applications; those contemporaneously realized with the theorems are presented in AB2] and AS3. Here is a small sample. Let $X$ be a connected closed complex manifold with $H^{q}\left(X ; \mathcal{O}_{X}\right)=0$ for $q>0$. Then any holomorphic map $f: X \rightarrow X$ has a fixed point. In a different direction, let $G$ be a compact connected Lie group, and let $T \subset G$ be a maximal torus. Then Theorem 5.4 applied to the action of a generic element of $T$ on a holomorphic line bundle $\mathscr{L} \rightarrow G / T$ over the flag manifold leads to the Weyl character formula. On a compact Riemannian manifold $X$ one deduces strong consequences of the generalized Lefschetz theorems using the signature operator. For example, Atiyah, Bott, and Milnor prove that two $h$-cobordant lens spaces are isometric. Some number theoretic aspects of the Lefschetz formula for the signature operator are described in the Atiyah-Bott and Atiyah-Singer papers. These ideas are elaborated much further by Hirzebruch and Zagier [HZ], who explore this Lefschetz signature theorem not only for lens spaces, but also for projective spaces, Brieskorn varieties, and algebraic surfaces. They find deep relations with cotangent sums, the Dedekind eta function, modular forms, and real quadratic fields, among other number theoretic objects of interest. This link between Lefschetz-type invariants and number theory is central to the developments in the 1970s, as we take up in $\$ 7$.

5.2. Index theorem on manifolds with boundary. As discussed in $₫ 3.3$, the study of linear elliptic equations traditionally takes place on a domain $\Omega \subset \mathbb{A}^{n}$ with smooth boundary, in which case elliptic boundary conditions need be imposed. ( $\mathbb{A}^{n}$ is the standard real affine $n$-dimensional space.) For the second-order Laplace operator (4.2) on $\bar{\Omega}$, the Dirichlet problem is the system of equations

$$
\begin{aligned}
\Delta u & =0, \\
\left.u\right|_{\partial \Omega} & =f
\end{aligned}
$$

for a prescribed function $f$ on $\partial \Omega$. The second equation in (5.6) is a local boundary condition for $\Delta$ : at each point $x \in \partial \Omega$ it depends only on $u(x)$. More generally, a local boundary condition may depend on a finite set of derivatives of $u$ at $x$. Lopatinski [Lo] gave a general criterion for a local boundary condition to be elliptic; see [Ho1, §20.1], for example.

Not all elliptic operators admit local elliptic boundary conditions. Consider the first-order $d$-operator on functions $u: \bar{\Omega} \rightarrow \mathbb{C}$ where $\Omega \subset \mathbb{C}$ is the unit disk. The kernel of $d$ consists of holomorphic functions on $\bar{\Omega}$, an infinite dimensional vector space, as expected in the absence of elliptic boundary conditions. For this operator there do not exist local elliptic boundary conditions: the Cauchy-Riemann equations on a disk are ill-posed. (As we will see in $\$ 7.2$, there are global boundary conditions. Also, if we consider the $d$-operator on sections of a vector bundle - as, for example, when studying deformations of holomorphic disks in a symplectic manifold with boundary on a Lagrangian submanifold - then there do exist local boundary conditions.) The topological nature of the existence question for local elliptic boundary conditions was brought out in work of Atiyah and Bott AB3, A2 . Again, $K$-theory is the natural home for the obstruction. In fact, their work led to a new proof of the Bott periodicity theorem [AB4]. Let $X$ be a compact manifold with boundary, and suppose $\sigma \in K^{0}\left(T^{*} X, T^{*} X \backslash 0\right)$ is an elliptic symbol class. The obstruction 
to local elliptic boundary conditions is the restriction of $\sigma$ to $K^{0}\left(\left.T^{*} X\right|_{\partial X}\right)$, and if the obstruction vanishes, then local elliptic boundary conditions lift $\sigma$ to an appropriate relative $K$-theory group. Atiyah, Bott, and Singer prove an index theorem for elliptic operators with local elliptic boundary conditions. The topological index easily extends to the lifted relative elliptic symbol, and the topological index of that lifted symbol computes the analytic index of the elliptic boundary value problem.

5.3. Real elliptic operators. Consider the operator $D=d / d x$ acting on realvalued functions on $\mathbb{R} / 2 \pi \mathbb{Z}$, i.e., on $2 \pi$-periodic functions $u: \mathbb{R} \rightarrow \mathbb{R}$. Integration by parts shows that $D$ is (formally) skew-adjoint. It is an example of a real Dirac operator. A closely related Dirac operator is $D^{\prime}=d / d x$ acting on twisted functions on the circle, or equivalently $u: \mathbb{R} \rightarrow \mathbb{R}$ which satisfy $u(x+2 \pi)=-u(x)$ for all $x \in \mathbb{R}$. Observe ker $D \cong \mathbb{R}$ consists of constant functions, whereas ker $D^{\prime}=0$. A skew-adjoint operator has integer index zero in the sense of (3.12). But for skew-adjoint Fredholm operators $P$, the mod $2 \operatorname{dimension} \operatorname{dim} \operatorname{ker} P(\bmod 2)$ is a deformation invariant: skew-adjointness implies that nonzero eigenvalues move in pairs as $P$ deforms, so in particular $\operatorname{dim} \operatorname{ker} P$ can jump only by even numbers 12 For the Dirac operators $D, D^{\prime}$ on the circle, this $\bmod 2$ index distinguishes the two spin structures, which we have rendered here in a concrete form.

The symbol $p(x, \xi)$ of a real elliptic operator $P$ on $\mathbb{R}^{n}$, as a function of $\xi \in \mathbb{R}^{n *}$, is essentially the Fourier transform of a real-valued function; see (4.14). Thus $p$ is a complex-valued function which satisfies the reality condition

$$
p(x,-\xi)=\overline{p(x, \xi)} .
$$

The global symbol $\sigma(P)$ on a manifold $X$ is a map of complex vector bundles (3.11), and the reality condition (5.7) globalizes to a complex conjugate isomorphism of the vector bundles which covers the (-1)-involution on $T^{*} X$ and commutes with $\sigma(P)$. This idea led Atiyah to develop "Real $K$-theory" [A3]; see [Seg4, §8]. Building on this, Atiyah and Singer AS5] proved an index theorem for real elliptic operators. This index theorem covers mod 2 indices, such as the invariant on $S^{1}$ mentioned above, and other cases as well. For example, the natural integer invariant of the real Dirac operator in dimension 4 is one-half the integer index, which leads to an analytic proof of a theorem of Rokhlin Roh]: the signature of a closed spin 4-manifold is divisible by 16 . These real index theorems are illuminated by Clifford algebras, as explained in 5.4 .

Remark 5.8. By design, the $K$-theory proof $(\$ 4.5)$ is suited to prove generalizations of Theorem 4.18, such as the real index theorem. For the mod 2 indices I am unaware of other proofs, whereas for integer indices there are other approaches, such as the heat equation methods we survey in $\$ 6$.

5.4. Index theorems for Clifford linear operators. Let $X$ be a Riemannian spin manifold of dimension $n$, and let $\operatorname{Spin}(X) \rightarrow X$ be the principal $\operatorname{Spin}_{n}$-bundle which encodes the Riemannian spin structure; see \$4.1. Recall the Clifford algebras Cliff $_{ \pm n}$ with generators $\gamma^{1}, \ldots, \gamma^{n}$ and relations

$$
\gamma^{i} \gamma^{j}+\gamma^{j} \gamma^{i}= \pm 2 \delta^{i j}=\left\{\begin{array}{rl} 
\pm 2, & i=j ; \\
0, & i \neq j,
\end{array} \quad 1 \leq i, j \leq n .\right.
$$

\footnotetext{
${ }^{12}$ The spectrum of a Fredholm operator need not be discrete; still, the assertion about the kernel is valid.
} 
Use the "mixing construction" to form the vector bundle

$$
S_{X}=\operatorname{Spin}(X) \times_{\operatorname{Spin}_{n}} \operatorname{Cliff}_{+n} \longrightarrow X,
$$

where $\operatorname{Spin}_{n} \subset$ Cliff $_{+n}$ acts on Cliff $+n$ by left multiplication. The fibers of $S_{X} \rightarrow X$ are $\mathbb{Z} / 2 \mathbb{Z}$-graded right Cliff $_{+n}$-modules, since right and left multiplication commute. Then since Cliff $_{-n}$ is the $\left(\mathbb{Z} / 2 \mathbb{Z}\right.$-graded) opposite algebra to Cliff ${ }_{+n}$, the fibers of $S_{X} \rightarrow X$ are $\mathbb{Z} / 2 \mathbb{Z}$-graded left Cliff $_{-n}$-modules. Furthermore, $S_{X} \rightarrow X$ inherits a covariant derivative from the Levi-Civita connection. The Dirac operator (4.6) acts on sections of $S_{X} \rightarrow X$, where we use the embedding $\mathbb{R}^{n} \hookrightarrow \mathrm{Cliff}_{+n}$ as the span of $\gamma^{1}, \ldots, \gamma^{n}$ to define Clifford multiplication. Then $D_{X}$ is a skew-adjoint Fredholm operator (on suitable Sobolev spaces), it is odd with respect to the grading, and it graded-commutes with the left Cliff $_{-n}$-action.

\section{Remark 5.11.}

(1) This Clifford linear Dirac operator appears in [LM, §II.7], attributed to Atiyah and Singer.

(2) As for any irreducible representation, the spin representations $\mathbb{S}^{0}, \mathbb{S}^{1}$ in $\$ 4.1$ are only determined up to tensoring with a line, as already remarked there. By contrast, the construction of $S_{X} \rightarrow X$ and $D_{X}$ are canonical for Riemannian spin manifolds. We have traded the irreducible representations $\mathbb{S}^{0}, \mathbb{S}^{1}$ for the canonical representation Cliff $+n$ equipped with a canonical commuting algebra of operators.

(3) One can complexify Cliff $+n$ and so $S_{X} \rightarrow X$ to construct a canonical complex Dirac operator, but the real Dirac operator contains more refined information.

(4) If $V \rightarrow X$ is a real vector bundle with covariant derivative, we can form a twisted Dirac operator $D_{X}(V)$ on sections of $S_{X} \otimes V \rightarrow X$; it too commutes with the left Cliff $_{-n}$-action.

The kernel and cokernel of $D_{X}(V)$ are finite-dimensional Cliff ${ }_{-n}$-modules, so by [ABS] their formal difference - the index - represents an element of the real $K$ theory group $K O^{-n}(\mathrm{pt})$. This is the analytic pushforward of $V$. The spin structure orients the map $f: X \rightarrow$ pt for real $K$-theory (see (2.27) for the analogous situation in complex $K$-theory) and the topological pushforward is $f_{!}[V] \in K O^{-n}(\mathrm{pt})$. The index theorem for Dirac operators asserts the equality of these pushforwards. The $K O$-groups of a point are computed from Bott periodicity. Let $O_{\infty}$ be the stable orthogonal group. Then for $n \geq 1$,

$$
K O^{-n}(\mathrm{pt}) \cong \pi_{n-1} O_{\infty} \cong\left\{\begin{array}{lll}
\mathbb{Z}, & n \equiv 0 & (\bmod 8) ; \\
\mathbb{Z} / 2 \mathbb{Z}, & n \equiv 1 & (\bmod 8) ; \\
\mathbb{Z} / 2 \mathbb{Z}, & n \equiv 2 & (\bmod 8) ; \\
0, & n \equiv 3 & (\bmod 8) ; \\
\mathbb{Z}, & n \equiv 4 & (\bmod 8) ; \\
0, & n \equiv 5 & (\bmod 8) ; \\
0, & n \equiv 6 & (\bmod 8) ; \\
0, & n \equiv 7 & (\bmod 8) .
\end{array}\right.
$$

$\left(K O^{0}(\mathrm{pt}) \cong \mathbb{Z}\right.$ is also correct.) The $\bmod 2$ index for $n=1$ discussed in $\$ 5.3$ is a special case. 


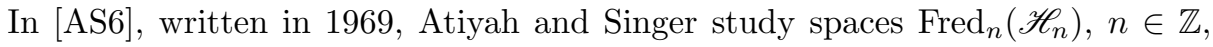
of odd skew-adjoint Fredholm operators acting on real and complex $\mathbb{Z} / 2 \mathbb{Z}$-graded Hilbert spaces $\mathscr{H}_{n}$ equipped with a $\mathrm{Cliff}_{n}$ action; the operators graded-commute with Cliff $_{n}$. The Clifford linear Dirac operator is an example. The main result is that the $\operatorname{spaces} \operatorname{Fred}_{n}\left(\mathscr{H}_{n}\right)$ form a spectrum in the sense of homotopy theory, and Atiyah and Singer identify it with the $K O$ - and $K$-theory spectra in the real and complex cases, respectively. This generalizes the relationship between $K$-theory and Fredholm operators in earlier work of Jänich [J]. It also gives an alternative construction of the analytic index of the Clifford linear Dirac operator. Finally, the periodicity of Clifford algebras $\mathrm{ABS}$ ] leads to another proof of Bott periodicity; see also Kar,Wo.

5.5. Families index theorem. Recall that Grothendieck, in his Riemann-Roch Theorem 2.14 considers proper morphisms $f: X \rightarrow S$, not just a single variety $X \rightarrow$ pt. Similarly, Atiyah and Singer [AS4] generalize Theorem 4.18 to proper fiber bundles $f: X \rightarrow S$ equipped with a family $P$ of elliptic (pseudo)differential operators along the fibers. On suitable function spaces, $P$ is a continuous family of Fredholm operators parametrized by $S$, and the analytic index is the homotopy class of this family. Since Fredholm operators form a classifying space for $K$-theory [J, A4, AS6, the analytic index lies in $K^{\bullet}(S)$. The topological index construction, executed in the fiber bundle $f$, also leads to an element of $K^{\bullet}(S)$. The index theorem for families is the equality of the analytic and topological indices.

There is also a families version of the Clifford linear story of $\$ 5.4$, which we illustrate.

Example 5.13. Let $f: X \rightarrow S$ be a proper fiber bundle with fibers of odddimension $n$. Suppose a relative Riemannian spin structure 13 is also given. In complex $K$-theory there is a pushforward

$$
f_{!}: K^{0}(X) \longrightarrow K^{-n}(S) .
$$

If $V \rightarrow X$ is a complex vector bundle with covariant derivative, we form the family $D_{X / S}(V)$ of complex Clifford linear Dirac operators parametrized by $S$. The analytic index is the homotopy class of the map $S \rightarrow$ Fred $_{-n}$ given by the Dirac operators, where Fred $_{-n}$ is the space of Fredholm operators introduced at the end of $\$ 5.4$ a classifying space for $K^{-n}$. The index theorem asserts that the analytic index equals $f_{!}[V]$. The "lowest piece" of the index is captured by composing with a natural map

$$
K^{-n}(S) \longrightarrow H^{1}(S ; \mathbb{Z})
$$

which may be considered a determinant map. Recalling that a class in $H^{1}(S ; \mathbb{Z})$ is determined by its periods on maps $\varphi: S^{1} \rightarrow S$, in other words by its values on $\varphi_{*}\left[S^{1}\right] \in H_{1}(S)$, since $H^{1}(S ; \mathbb{Z})$ is torsion-free, we compute the image of ind $D_{X / S}(V)$ under (5.15) by base changing via $\varphi$ to a fiber bundle $X_{\varphi} \rightarrow S^{1}$ equipped with a vector bundle $V_{\varphi} \rightarrow X_{\varphi}$. Then $X_{\varphi}$ is an even-dimensional closed Riemannian spin manifold, and the integer period we seek is the numerical index ind $D_{X_{\varphi}}\left(V_{\varphi}\right) \in \mathbb{Z}$.

\footnotetext{
${ }^{13} \mathrm{~A}$ relative spin structure is a spin structure on the vertical tangent bundle $T(X / S) \rightarrow X$. A relative Riemannian structure is an inner product on this vector bundle together with a horizontal distribution on the fiber bundle $f$.
} 
Remark 5.16.

(1) The family of Clifford linear Dirac operators $D_{X / S}(V)$ gives rise to a family of self-adjoint Dirac operators on an ungraded Hilbert space, a formulation which appears more frequently; see [APS3, §3], for example.

(2) A cohomology class in $H^{1}(S ; \mathbb{Z})$ is a homotopy class of maps $S \rightarrow \mathbb{R} / \mathbb{Z}$. A geometric invariant of the family $D_{X / S}(V)$ - the Atiyah-Patodi-Singer $\eta$-invariant - promotes this homotopy class to a specific map, as we will see in $\$ 7.3$,

(3) Although we stated these topological constructions for Dirac operators, they generalize in various ways to families of elliptic pseudodifferential operators.

A situation in which one encounters a family of linear elliptic equations is linearization of solutions to a nonlinear elliptic equation. The abstract setup is a nonlinear Fredholm map $F: \mathscr{B} \rightarrow \mathscr{C}$ between infinite-dimensional Hilbert manifolds. Sometimes $F$ is equivariant for the action of an infinite-dimensional Lie group $\mathscr{G}$ and is only Fredholm modulo $\mathscr{G}$. One is then interested in the moduli space, or stack, $\mathscr{M}=F^{-1}(c) / \mathscr{G}$ for some $c \in \mathscr{C}$. If $F$ is a nonlinear elliptic operator on a closed manifold, mapping between function spaces, then its linearizations $d F_{b}$ at $b \in F^{-1}(c)$ fit together to a family of linear elliptic operators parametrized by $\mathscr{B}$. The index of this family computes the (virtual) tangent bundle to $\mathscr{M}$ and yields useful information about $\mathscr{M}$. This general plan is used by Atiyah, Hitchin, and Singer $\mathrm{AHS}$ to investigate the instanton equations on a 4-manifold (see Donaldson's paper [Do, §2] in this volume) and it has been used since in many other problems in geometric analysis.

Remark 5.17. The image of the families index under the Chern character ch: $K^{\bullet}(S) \rightarrow H^{\bullet}(S ; \mathbb{Q})$ is a cruder invariant than the $K$-theory index, but often it contains information of interest. It can be computed by a topological analogue of (2.15), and it is accessible via heat equation methods Bi2, whereas the more powerful $K$-theory index is not, as far as we know.

The Atiyah-Hitchin-Singer work is the first of many applications of the families index theorem to quantum field theory and string theory, and to mathematical problems arising from that physics. We take up an additional example in 88 .

5.6. Coverings and von Neumann algebras. As should be clear by now, Atiyah used the index theorem as a launching pad for mathematical adventures in many directions. One which proved particularly fruitful involves von Neumann algebras [A5]. Atiyah [A1] comments:

In particular I learnt from Singer, who had a strong background in functional analysis, about von Neumann algebras of type II with their peculiar real-valued dimensions. We realized that $K$-theory and index theory could be generalized in this direction, but it was not clear at first if such a generalization would really be of any interest. However in one particularly simple case, that of a manifold with an infinite fundamental group, it became clear that the ideas of von Neumann algebras were quite natural and led to concrete non-trivial results. This was the content of my talk [A5] at the meeting in honour of Henri Cartan. Since I was not an expert on 
von Neumann algebras I attempted in this presentation to give a simple, elementary and essentially self-contained treatment of the results. Later on in the hands of Alain Connes, the world expert on the subject, these simple ideas were enormously extended and developed into a whole theory of linear analysis for foliations.

And in the hands of Connes, Kasparov, and many others into index theory and $K$-theory for $C^{*}$-algebras. An influential conference talk indeed!

The situation in A5] is an unramified Galois covering $\pi: \widetilde{X} \rightarrow X$ with Galois group $\Gamma$ acting freely on $\widetilde{X}$, and a $\Gamma$-invariant elliptic operator $\widetilde{D}$ on $\widetilde{X}$. There is an induced elliptic operator $D$ on $X$, and we assume $X$ is compact. For example, $X$ could be a closed Riemann surface and $\widetilde{X}$ its universal cover which, if the genus of $X$ is $\geq 2$, is isomorphic to the unit disk $\Omega$. If $\widetilde{D}$ is the $d$-operator, then ker $\widetilde{D}$ is the infinite-dimensional space of holomorphic functions on $\Omega$. In general, if $\Gamma$ is infinite then $\operatorname{ker} \widetilde{D}$ and coker $\widetilde{D}$ are infinite dimensional, whereas $\operatorname{ker} D$ and coker $D$ are finite dimensional, the latter since $X$ is assumed compact. So ind $D$ is well-defined. Atiyah introduces a $\Gamma$ invariant measure on $\widetilde{X}$ and the von Neumann algebra $\mathfrak{A}$ of bounded linear operators on $L^{2}\left(\widetilde{X}, \widetilde{E}^{0}\right)$ for $\widetilde{E}^{0} \rightarrow \widetilde{X}$ the vector bundle on whose sections $\widetilde{D}$ is defined. Orthogonal projection onto ker $\widetilde{D}$ lies in the von Neumann algebra, and its von Neumann trace, a real number, is defined to be the $\Gamma$ dimension of ker $\widetilde{D}$. Repeating for coker $\widetilde{D}$, Atiyah defines a real-valued index $\operatorname{ind}_{\Gamma} \widetilde{D}$.

Theorem 5.18 (Atiyah [A5]). $\operatorname{ind}_{\Gamma} \widetilde{D}=$ ind $D$.

Atiyah's account of this theorem, as stated earlier, was a catalyst for index theory on noncompact spaces, singular spaces, and beyond.

\section{Heat equation proof}

Beginning in the late 1960s the expanding circle of ideas emanating from the basic Atiyah-Singer index theorem took a more analytic turn. The focus shifted beyond the kernel of elliptic operators to include higher eigenvalues. This led first to a local version of the index theorem and then to local geometric invariants (as opposed to global topological invariants). We treat the former in this section and the latter in the next.

We begin in 6.1 with two basic constructs to collate higher eigenvalues into a single function: the $\zeta$-function and the trace of the heat operator. (They are analogues of basic objects in analytic number theory.) The local index theorem, proved first in special cases by Vijay Patodi and then in general by Peter Gilkey, is the subject of 86.2 . This work was completed in the early 1970s. In the 1980s several new proofs of the local index theorem led to a deeper understanding of the origins of the $\hat{A}$-genus in the index formula for Dirac operators. In 6.3 we briefly summarize these contributions by Ezra Getzler, Edward Witten, Jean-Michel Bismut, Nicole Berline, Michele Vergne, and of course Michael Atiyah.

6.1. Heat operators, zeta functions, and the index. Let $\Delta$ be a nonnegative self-adjoint operator on a Hilbert space $\mathscr{H}$. We seek to define the heat operator

$$
H_{t}=e^{-t \Delta}, \quad t \in \mathbb{R}^{>0},
$$

and the $\zeta$-function

$$
\zeta_{\Delta}(s)=\operatorname{Tr} \Delta^{-s}, \quad s \in \mathbb{C} .
$$


Both are well-defined if $\mathscr{H}$ is finite dimensional, and they are related by the Mellin transform

$$
\operatorname{Tr} \Delta^{-s}=\frac{1}{\Gamma(s)} \int_{0}^{\infty} \frac{d t}{t} t^{s} \operatorname{Tr}\left(e^{-t \Delta}\right)
$$

If $\Delta$ is a nonnegative self-adjoint second-order elliptic operator on a closed manifold $X$, then the heat operator $H_{t}$ in (6.1) exists by basic elliptic theory. It is a smoothing operator: $H_{t}$ maps distributions to smooth functions. For example, if $\delta_{y}$ is the Dirac $\delta$-distribution at $y \in X$, then 14

$$
h_{t}(x, y)=\left(e^{-t \Delta} \delta_{y}\right)(x), \quad x \in X,
$$

is a smooth function of $t, x, y$ called the heat kernel. If $\Delta$ is the scalar Laplace operator attached to a Riemannian metric on $X$ - the Laplace-Beltrami operatorthen intuitively $h_{t}(x, y)$ is the amount of heat at $x$ after time $t$ given an initial distribution $\delta_{y}$ of heat. Heat flows with infinite propagation speed and instantly diffuses: $h_{t}(x, y)>0$ for all $t, x, y$. The properties of physical heat flow inform intuition about the large and small time behavior of the heat operator of a general nonnegative self-adjoint second-order differential operator $\Delta$ acting on sections of a vector bundle $E \rightarrow X$. As $t \rightarrow \infty$ the heat operator $e^{-t \Delta}$ converges (in the uniform topology) to projection onto ker $\Delta$. As $t \rightarrow 0$ the heat operator converges (in the strong operator topology) to the identity operator. A more precise version of the small $t$ behavior is the subject of an influential 1948 paper of Minakshisundarum and Pleijel $\mathrm{MP}$ in case $\Delta$ is the Laplace-Beltrami operator; Seeley [Se4] extends their results to more general elliptic pseudodifferential operators. As $t \rightarrow 0$ the heat kernel $h_{t}(x, y)$ converges exponentially to zero if $x \neq y$, and on the diagonal there is an asymptotic expansion

$$
h_{t}(x, x) \sim t^{-n / 2} \sum_{k=0}^{\infty} A_{k}(x) t^{i} \quad \text { as } t \rightarrow 0,
$$

where $A_{k}$ are smooth functions on $X$. For $x \in X$, the value of $A_{k}(x) \in$ End $E_{x}$ depends only on a finite jet of the total symbol of the differential operator $\Delta$ at $x$. If $\Delta$ is canonically associated to a Riemannian metric, then $A_{k}(x)$ depends on a finite jet of the metric at $x$; the order of the jet grows with $k$.

For elliptic operators $\Delta$ on compact manifolds of the type discussed in the previous paragraph, the $\zeta$-function (6.2) exists and $\zeta_{\Delta}$ is a holomorphic function of $s$ for $\operatorname{Re}(s)>0$. The asymptotic expansion (6.5) of the heat kernel $h_{t}$ is equivalent, via the Mellin transform (6.3), to a meromorphic extension of $\zeta_{\Delta}$ to the entire complex $s$-line, which in fact is what is proved in [MP, Se4].

Now suppose $P: C^{\infty}\left(X, E^{0}\right) \rightarrow C^{\infty}\left(X, E^{1}\right)$ is a first-order elliptic operator on a closed $n$-manifold $X$ equipped with complex vector bundles $E^{0}, E^{1} \rightarrow X$. Assume metrics everywhere so that the formal adjoint $P^{*}: C^{\infty}\left(X, E^{1}\right) \rightarrow C^{\infty}\left(X, E^{0}\right)$ is defined; it too is a first-order elliptic operator. Then

$$
\text { ind } P=\operatorname{dim} \operatorname{ker} P^{*} P-\operatorname{dim} \operatorname{ker} P P^{*} \text {, }
$$

and each of $P^{*} P, P P^{*}$ is a nonnegative self-adjoint second-order elliptic differential operator. For $\lambda \geq 0$, let $\mathscr{E}_{\lambda}^{0} \subset C^{\infty}\left(X, E^{0}\right)$ be the $\lambda$-eigenspace of $P^{*} P$, and let

\footnotetext{
${ }^{14}$ Our notation assumes $\Delta$ is an operator acting on functions. A small modification incorporates vector bundles.
} 
$\mathscr{E}_{\lambda}^{1} \subset C^{\infty}\left(X, E^{1}\right)$ be the $\lambda$-eigenspace of $P P^{*}$. Then for $\lambda>0$,

$$
\left.P\right|_{\mathscr{E}_{\lambda}^{0}}: \mathscr{E}_{\lambda}^{0} \longrightarrow \mathscr{E}_{\lambda}^{1}
$$

is an isomorphism. Therefore, for any function $\chi: \mathbb{R}^{\geq 0} \rightarrow \mathbb{R}^{\geq 0}$ such that $\chi(0)=1$ and $\chi(\lambda) \searrow 0$ sufficiently rapidly as $\lambda \rightarrow \infty$,

$$
\text { ind } P=\sum_{\lambda \in \operatorname{spec} P^{*} P} \chi(\lambda)-\sum_{\lambda \in \operatorname{spec} P P^{*}} \chi(\lambda) \text {. }
$$

For $\chi(\lambda)=\lambda^{-s}$ with $\operatorname{Re}(s)>>0$, we obtain a formula for the index which appears in Atiyah and Bott's paper [AB1, $\S 8$ ]:

$$
\text { ind } P=\operatorname{Tr} \zeta_{P^{*} P}(s)-\operatorname{Tr} \zeta_{P P^{*}}(s) \text {. }
$$

In fact (6.9) holds for all $s \in \mathbb{C}$, due to the meromorphic continuation of zeta functions. Atiyah and Bott note that $s=0$ is a particularly good argument in view of explicit integral formulas [Se4] in terms of the symbol of $P$. (Another motivation for setting $s=0$ : for an operator $P: \mathscr{H}^{0} \rightarrow \mathscr{H}^{1}$ between finite-dimensional Hilbert spaces, the value at $s=0$ is $\operatorname{dim} \mathscr{H}^{0}-\operatorname{dim} \mathscr{H}^{1}$.) But while the explicit formulas are local, they involve high derivatives of the symbol, whereas the characteristic class formula (4.10) for the index only involves a few derivatives when written in terms of Chern-Weil polynomials of the curvature. It is this mismatch which remained a mystery for several years.

In place of $\zeta$-functions, the trace of the heat kernel is commonly used in (6.8). This corresponds to $\chi(\lambda)=e^{-t \lambda}, t>0$. Then for all $t>0$, we have

$$
\text { ind } P=\operatorname{Tr} e^{-t P^{*} P}-\operatorname{Tr} e^{-t P P^{*}} \text {. }
$$

In fact, one can prove the right hand side is constant in $t$ by differentiation, and evaluation as $t \rightarrow \infty$ reproduces (6.10). On the other hand, let $t \rightarrow 0$ and use the asymptotic expansion (6.5) to obtain

$$
\text { ind } P=\int_{X} \operatorname{tr}\left[A_{n / 2}^{0}(x)-A_{n / 2}^{1}(x)\right]|d x|,
$$

where $A_{k}^{0}(x), A_{k}^{1}(x)$ are the heat coefficients for $P^{*} P, P P^{*}$ acting on the vector spaces $E_{x}^{0}, E_{x}^{1}$, respectively. As is true for the $\zeta$-function, the formulas for $A_{n / 2}^{i}(x)$ involve many derivatives of the symbol of $P$ at $x$, so seem inaccessible as a means of proving the index formula.

6.2. The local index theorem. For the Laplace-Beltrami operator $\Delta$ on an $n$ dimensional Riemannian manifold $X$, the first coefficient $A_{0}$ in (6.5) is the constant function $1 /(4 \pi)^{n / 2}$. This reflects the solution to the classical heat equation in Euclidean space, and it implies Weyl's law for the asymptotic growth of the eigenvalues of $\Delta$, which only depends on $n$ and $\operatorname{Vol}(X) \sim \int_{X} A_{0}(x)|d x|$. Weyl's law, which does not depend on the heat kernel expansion, was one motivation for Mark Kac $[\mathrm{K}$ to ask in 1966: To what extent do the eigenvalues of $\Delta$ determine the Riemannian manifold $X$ ? Kac focused on domains in the Euclidean plane $\mathbb{E}^{2}$, though the more general question is implicit. McKean and Singer [MS immediately took this up, and they determined the next few coefficients $A_{1}, A_{2}$ in the heat kernel expansion, thereby proving a conjecture of Kac and Pleijel. (There are contemporaneous independent results by de Bruijn, Arnold, and Berger.) In particular, $A_{1}$ is a multiple of the scalar curvature. For $n=2$, McKean and Singer observe a cancellation 
which holds at each point of $X$, and they conjecture a similar result in all dimensions. Namely, let $\Delta^{(q)}$ denote the Laplace operator on differential $q$-forms, let $h_{t}^{(q)}$ denote the associated heat kernel, and let $A_{k}^{(q)}$ denote the heat coefficients. The McKean-Singer conjecture is that for all $x \in X$ the limit

$$
\lim _{t \rightarrow 0} \sum_{q=0}^{n}(-1)^{q} \operatorname{tr} h_{t}^{(q)}(x, x)
$$

exists and, furthermore, for $n$ even it equals the Gauss-Bonnet-Chern integrand which integrates to the Euler number of $X$. From (6.5) the existence of the limit is equivalent to the cancellation

$$
\sum_{q=0}^{n}(-1)^{q} \operatorname{tr} A_{k}^{(q)}(x)=0, \quad k<\frac{n}{2}, \quad x \in X .
$$

The alternating sum for $k=n / 2$ equals the limit (6.12). McKean and Singer prove that the limit exists and vanishes for $n$ odd, and they compute the limit for $n=2$.

In 1970 Patodi [P1] proved the McKean-Singer conjecture via a virtuoso direct computation. Patodi immediately [P2] applied his methods to prove the RiemannRoch Theorem 2.7 for Kähler manifolds. A few years later, Gilkey used different methods in his $\mathrm{PhD}$ thesis Gi1 - scaling plays a crucial role - and proved the corresponding theorem for twisted signature operators. By standard topological arguments this implies the Atiyah-Singer Index Theorem 4.9, see \$4.2. Subsequently, Atiyah, Bott, and Patodi $\mathrm{ABP}$ gave a proof of Gilkey's theorem and of the resulting proof of the index theorem. We recount Gilkey's main result.

Gilkey investigates differential forms built canonically from a Riemannian metric. Using modern terminology to economize, let $\mathbf{M a n}_{n}$ be the category of smooth $n$ manifolds and local diffeomorphisms. Consider the functors (sheaves)

$$
\begin{gathered}
\text { Met: } \operatorname{Man}_{n}^{\mathrm{op}} \longrightarrow \text { Set } \\
\Omega^{q}: \mathbf{M a n}_{n}^{\mathrm{op}} \longrightarrow \text { Set },
\end{gathered}
$$

where if $M$ is a smooth $n$-manifold, then $\operatorname{Met}(M)$ is the set of Riemannian metrics on $M$ and $\Omega^{q}(M)$ is the set of differential $q$-forms. We seek natural transformations

$$
\omega: \text { Met } \longrightarrow \Omega^{q} \text {. }
$$

Roughly speaking, these are assignments of differential forms to Riemannian metrics covariant under coordinate changes. More poetically, they are differential forms on Met. Even for $q=0$, the classification problem is intractable: any smooth function of the scalar curvature gives a natural function of the Riemannian metric. Now introduce scaling. We say $\omega$ is homogeneous of weight $k$ if

$$
\omega\left(\lambda^{2} g\right)=\lambda^{k} \omega(g) \quad \text { for all } \lambda \in \mathbb{R}^{>0} .
$$

We say $\omega$ is regular if in any local coordinate system $x^{1}, \ldots, x^{n}$ it takes the form

$$
\omega(g)(x)=\sum_{I} \sum_{\alpha}^{\text {finite }} \sum_{i, j=1}^{n} \omega_{I, \alpha}^{i, j}(x) \frac{\partial^{|\alpha|} g_{i j}}{\partial x^{\alpha_{1}} \cdots \partial x^{\alpha_{n}}} d x^{i_{1}} \wedge \cdots \wedge d x^{i_{q}},
$$

where $I=\left(i_{1}, \ldots, i_{q}\right)$ with $1 \leq i_{1}<\cdots<i_{q} \leq n$, and $\alpha=\left(\alpha_{1}, \ldots, \alpha_{n}\right)$ with $\alpha_{k} \in \mathbb{Z}^{\geq 0}$. The $\omega_{I, \alpha}^{i j}$ are smooth functions. Crucially, only a finite set of $\alpha$ appears. 
Theorem 6.18 (Gilkey Gi1]). A natural differential form (6.15) which is regular and homogeneous of nonnegative weight is a polynomial in the Chern-Weil forms of the Pontrjagin classes.

The nonzero forms have weight zero.

The proof of Gilkey's theorem, Theorem 6.18 in [ABP, uses Weyl's theorem [W] on invariants of the orthogonal group. Atiyah, Bott, and Patodi apply Theorem 6.18 to the signature operator $P$ on a Riemannian manifold $X$. Resuming the notation of $₫ 6.1$, the vanishing of positive weight forms implies

$$
\operatorname{tr}\left[A_{k}^{0}(x)-A_{k}^{1}(x)\right]=0, \quad k<\frac{n}{2}, \quad x \in X .
$$

This cancellation result implies, as in (6.13), the existence of

$$
\lim _{t \rightarrow 0}\left[\operatorname{tr} h_{t}^{0}(x, x)-\operatorname{tr} h_{t}^{1}(x, x)\right],
$$

and Theorem 6.18 tells that the limit is a polynomial in Pontrjagin forms. The precise polynomial - the $L$-genus (2.10) - is determined as in Hirzebruch's original proof by computing enough examples. The road from here to the global index theorem follows established lines. It is the local index theorem-the existence and identification of the limit (6.20) - which leads to future developments.

6.3. Postscript: Whence the $\hat{A}$-genus? Different conceptual understandings of the cancellation (6.19) and of the limiting value (6.20) were achieved in the first half of the 1980s. The setting is (generalized) Dirac operators, where basic properties of Clifford algebras yield the cancellation. The limit is the Chern-Weil $\hat{A}$-form, a polynomial in the Pontrjagin forms, whose appearance is derived from various sources. In these works the $\hat{A}$-genus appears by direct argument. We give a brief resumé.

One route to the $\hat{A}$-genus passes through Mehler's formula for the heat kernel of the harmonic oscillator [GJ, p. 19], which Getzler [Ge2 employs in his proof of the local index theorem. He uses a homothety which not only scales time and space, but also scales the Clifford algebra variables in the Dirac operator (4.6). His technique was in part inspired by contemporary physics proofs of the index theorem [Wi1, Ag, FW, Ge1 using supersymmetric quantum mechanics.

At a conference in honor of Laurent Schwartz, Atiyah A6 exposed Witten's idea to derive the index theorem by applying the Duistermaat-Heckman exactness of stationary phase theorem $[\mathrm{DH}]$ to the free loop space of a compact Riemannian manifold. (During that period Atiyah-Bott AB5 placed the DuistermaatHeckman result in the context of localization in equivariant cohomology.) In this proof the $\hat{A}$-genus enters by regularizing a certain infinite product, as it does in the supersymmetric quantum mechanics proof [Wi2, §1.2.4].

Inspired by Atiyah's account, Bismut Bi1 executed a proof of the index theorem using Wiener measure on loop space and Malliavin calculus. In this way he deals with integrals over loop space rigorously. The heat kernel is represented in terms of Wiener measure with the aid of Lichnerowicz's formula, which expresses the Dirac Laplacian in terms of the covariant Laplacian. The localization to point loops as $t \rightarrow 0$ is natural in this probabilistic approach. The variable $t$ represents the total time during which a Brownian path exists, and as the time tends to zero, only constant loops have a significant probability of occurring. The evaluation of the integral over these point loops is accomplished using a formula of Paul Lévy $[\mathrm{L}$, who 
considers a Brownian curve in the plane conditioned to close after time $2 \pi$. Then the characteristic function of the area $S$ enclosed by the random curve (expectation value of $e^{i z S}, z \in \mathbb{C}$ ) is $\pi z / \sinh \pi z$. This same calculation appears in Bismut's work, only there the curvature of $X$ replaces $z$, and once again the $\hat{A}$-genus is obtained.

The $\hat{A}$-genus arises quite differently in a proof of the index theorem due to Berline and Vergne $\mathrm{BV}$. Let $G$ be a Lie group with Lie algebra $\mathfrak{g}$. Then a standard formula in the theory of Lie groups asserts that the differential of the exponential map exp: $\mathfrak{g} \rightarrow G$ at $a \in \mathfrak{g}$ is

$$
d \exp _{a}=\frac{1-e^{-\operatorname{ad} a}}{\operatorname{ad} a}=J(\operatorname{ad} a)
$$

where the power series which defines $J$ is the multiplicative inverse of the power series which defines the Todd genus (2.5). It was a mystery whether the occurrence of the Todd genus in 6.21) is related to the index theorem. Berline and Vergne noticed that if $X$ is a Riemannian manifold, and $\mathrm{O}(X)$ the principal bundle of orthonormal frames, then the differential of the Riemannian exponential map on $\mathrm{O}(X)$ is given by a similar formula. Precisely, there is a natural isomorphism $T_{p} \mathrm{O}(X) \cong \mathbb{R}^{n} \oplus \mathfrak{o}(n)$ via the Levi-Civita connection, and the differential of the exponential map exp: $\mathbb{R}^{n} \oplus \mathfrak{o}(n) \rightarrow \mathrm{O}(X)$ at $p \in \mathrm{O}(X)$, evaluated on $a \in \mathfrak{o}(n)$, is

$$
\begin{aligned}
& \left.d \exp _{a}\right|_{\mathbb{R}^{n}}=\exp (-a) J\left(\left\langle\Omega_{p} / 2, a\right\rangle\right), \\
& \left.d \exp _{a}\right|_{\mathfrak{o}(n)}=J(\operatorname{ad} a) .
\end{aligned}
$$

In this formula the Riemann curvature $\Omega$, which takes values in $\mathfrak{o}(n)$, is contracted with $a$ using the Killing form. The result is a 2 -form, which can be identified as an element of $\mathfrak{o}(n)$. To prove the index theorem, Berline and Vergne work on the frame bundle $\mathrm{O}(X)$, not on the base $X$. To compensate for the introduction of extra degrees of freedom in the fiber direction, they must study the behavior of the heat kernel along the fiber. It is at this stage, in the small time limit, where (6.22) appears. Ultimately, that is how the $\hat{A}$-genus enters their proof.

\section{Geometric invariants of Dirac operators}

Up to this point index theory produced global topological invariants of elliptic operators; their natural home is topological $K$-theory. Beginning with the work Atiyah, Patodi, and Singer announced in 1973, index theory took a turn towards local differential geometric invariants 15 Furthermore, the focus shifted from general elliptic pseudodifferential operators to Dirac operators. Heat equation methods provide the fundamental tools to construct invariants.

The three papers APS1, APS2, APS3 of Atiyah, Patodi, and Singer introduce the $\eta$-invariant of a Dirac operator. Its definition is parallel to that of an $L$-function in analytic number theory. Their first main theorem, which we recount in $\$ 7.3$, is an index theorem for a Dirac operator on a compact Riemannian spin manifold with boundary. A key ingredient in the story are new global elliptic boundary conditions ( $(7.2)$; local elliptic boundary conditions are obstructed in most cases, as Atiyah and Bott had discovered a decade earlier. The $\eta$-invariant solves a problem which served as one motivation for their work, namely the computation of the

\footnotetext{
${ }^{15}$ The natural home for the geometric invariants is differential $K$-theory, but that is a more recent development and is beyond the scope of this article.
} 
signature defect, and this is our point of departure in \$7.1, The Atiyah-PatodiSinger papers contain many more important theorems, such as the index theorem for flat bundles, which we do not cover here.

Another view of the $\eta$-invariant is the subject of 7.4 Characteristic numbers of vector bundles over closed oriented manifolds - the integers obtained by pairing products of Chern and Pontrjagin classes of a vector bundle with the fundamental class of the base manifold - are primary integer-valued topological invariants. The associated $\mathbb{R} / \mathbb{Z}$-valued secondary differential geometric invariants had been introduced by Chern and Simons a few years prior. Similarly, integer-valued $K$-theory characteristic numbers, which by the index theorem are indices of Dirac operators, are primary topological invariants. The associated secondary differential geometric quantity is the Atiyah-Patodi-Singer $\eta$-invariant. The next geometric invariant of a Dirac operator, or family of Dirac operators, is the determinant. The underlying theory was developed in the 1980s, as we recount in 77.5 . It is an important ingredient in the application to physics we take up in $\$ 8$.

7.1. The signature defect. Recall the classical Gauss-Bonnet theorem. Let $X$ be a closed Riemannian 2-manifold, and let $K: X \rightarrow \mathbb{R}$ be its Gauss curvature. Then the Euler number of $X$ is the curvature integral

$$
\operatorname{Euler}(X)=\int_{X} \frac{K}{2 \pi} d \mu_{X}
$$

where $d \mu_{X}$ is the Riemannian measure. If now $X$ is compact with boundary, then there is a boundary contribution from the geodesic curvature $\kappa: \partial X \rightarrow \mathbb{R}$, namely

$$
\operatorname{Euler}(X)=\int_{X} \frac{K}{2 \pi} d \mu_{X}+\int_{\partial X} \frac{\kappa}{2 \pi} d \mu_{\partial X}
$$

If a neighborhood of $\partial X$ in $X$ is isometric to the cylinder $[0, \epsilon) \times \partial X$ with its product metric for some $\epsilon>0$, then the boundary term vanishes.

Now let $X$ be a closed oriented Riemannian 4-manifold. Hirzebruch's signature theorem, Theorem 2.11, implies

$$
\operatorname{Sign}(X)=\int_{X} \omega
$$

where $\omega$ is the Chern-Weil 4 -form of the rational characteristic class $p_{1} / 3$. If $X$ is compact with boundary, and even if we assume the Riemannian metric is a product near the boundary, which we do, formula (7.3) need not hold. Set $Y=\partial X$. Then the signature defect [A7, §10.3]

$$
\alpha(Y)=\operatorname{Sign}(X)-\int_{X} \omega
$$

depends only on the closed oriented Riemannian 3-manifold $Y$, as follows easily from (7.3). Atiyah, Patodi, and Singer APS1 argue that $\alpha$ is a smooth function of the Riemannian metric, is odd under orientation-reversal, and is not of the form $\int_{Y} \eta$ for some natural 3 -form $\eta$ in the metric (since the signature defect is not multiplicative under finite covers).

A concrete instance of the signature defect studied by Hirzebruch $[\mathrm{H} 4$ ] in the early 1970 s was a prime motivation for Atiyah, Patodi, and Singer. Let $K=\mathbb{Q}(\sqrt{d})$ be a real quadratic number field- $d \in \mathbb{Z}^{>1}$ is assumed square-free - and let $\mathcal{O} \subset K$ be 
the ring of integers. The two square roots of $d$ give two embeddings $K \hookrightarrow \mathbb{R}$, thus an embedding

$$
G=\mathrm{PSL}_{2}(\mathcal{O}) \hookrightarrow \mathrm{PSL}_{2}(\mathbb{R}) \times \mathrm{PSL}_{2}(\mathbb{R}) .
$$

Let $\mathbb{H}$ be the upper half-plane. The quotient

$$
X^{0}(K)=(\mathbb{H} \times \mathbb{H}) / G
$$

is a Hilbert modular surface. The group $G$ may act with finite stabilizers, i.e., $X^{0}(K)$ may be an orbifold which is not a smooth manifold. It is noncompact; $X^{0}(K)$ has a finite set of ends in bijection with the ideal class group of $K$. Truncate each end to construct a compact orbifold with boundary; each boundary component is a fiber bundle with base $S^{1}$ and fiber $S^{1} \times S^{1}$. Also, cut out a neighborhood of each orbifold point to obtain a smooth compact manifold $X(K)$ with boundary; the additional boundary components are lens spaces. The signature defect (7.4) at lens space boundaries was known [A7, §10.3] from the equivariant signature theorem (\$5.1). Hirzebruch [H4, p. 222] computed the signature defect at the other boundaries via a desingularization of the cusp singularity in the cone on the boundary. The formula is a simple expression in terms of a continued fraction associated to the singularity. On the other hand, Shimizu [Sh] introduced an $L$ function associated to the ideal class which corresponds to the end. Hirzebruch $\mathrm{H} 4$, p. 231] proves that the signature defect at the cusp is the value of that $L$-function at $s=1$, up to a numerical factor.

There is a generalization of this story to totally real number fields of arbitrary degree. For the general case Hirzebruch conjectured that the signature defect at a cusp singularity is again a value of the Shimizu $L$-function. This conjecture was proved independently by Atiyah, Donnelly, and Singer [ADS] and by Müller [Mu] in $1982-83$.

7.2. Global boundary conditions. To compute the signature defect (7.4) in general, it is natural to consider the signature operator (3.7) on a compact manifold with boundary. But we must impose an elliptic boundary condition, and for the signature operator the topological obstruction to local elliptic boundary conditions (5.2) is nonzero. Atiyah, Patodi, and Singer overcome this obstruction by a novel maneuver: they introduce global elliptic boundary conditions that exist for any generalized Dirac operator, including the signature operator. These global boundary conditions are now ubiquitous in the theory and applications of Dirac operators on manifolds with boundary.

As a first example, consider the $d$-operator on the closure of the unit disk $\Omega \subset \mathbb{C}_{z}$, as in 5.2 . The kernel consists of holomorphic functions on $\bar{\Omega}$; a dense subspace is the space of polynomials, the linear span of $\left\{z^{n}: n \in \mathbb{Z}^{\geq 0}\right\}$. This is an infinitedimensional vector space. An elliptic boundary condition must cut it down to a finite-dimensional subspace. Fix $a \in \mathbb{R} \backslash \mathbb{Z}^{\geq 0}$. Let $\mathscr{H}_{a}$ denote the subspace of smooth functions $u: \bar{\Omega} \rightarrow \mathbb{C}$ such that the Fourier expansion of $\left.u\right|_{\partial \Omega}$ has vanishing Fourier coefficient of $e^{i m \theta}$ if $m>a$, where we write $z=e^{i \theta}$ on $\partial \Omega$. Then the restriction of $\vec{d}$ to $\mathscr{H}_{a}$ has finite-dimensional kernel and cokernel, and it extends to a Fredholm operator on suitable Sobolev completions. In other words, restriction to $\mathscr{H}_{a}$ is an elliptic boundary condition. But because of the Fourier transform in its definition, it is not local; compare (5.6). 
This example generalizes to a Dirac operator $D_{X}$ on a compact Riemannian manifold $X$ with boundary. Assume the metric is a product near $Y=\partial X$, and so decompose

$$
D_{X}=\sigma \frac{\partial}{\partial t}+D_{Y}
$$

near the boundary. Here $t$ is the length coordinate on geodesics normal to $Y=\partial X$, the algebraic operator $\sigma$ is Clifford multiplication by $d t$, and $D_{Y}$ is a Dirac operator on $Y$. Then the operator $A_{Y}=\sigma^{-1} D_{Y}$ is self-adjoint. Let

$$
\bigoplus_{\lambda \in \operatorname{spec}\left(A_{Y}\right)} \mathscr{E}_{\lambda}
$$

be the spectral decomposition of spinors on $Y$. For each $a \in \mathbb{R} \backslash \operatorname{spec}\left(A_{Y}\right)$, the Atiyah-Patodi-Singer global boundary condition restricts to the subspace of spinors on $X$ whose restriction to $Y$ lies in the completion of $\bigoplus_{\lambda<a} \mathscr{E}_{\lambda}$. In fact, Atiyah, Patodi, and Singer choose $a=0$, as do we in what follows. If $0 \in \operatorname{spec}\left(A_{Y}\right)$, then one must take into account ker $D_{Y}$ separately, as in (7.10) below.

7.3. The Atiyah-Patodi-Singer $\eta$-invariant. With elliptic boundary conditions in hand, Atiyah, Patodi, and Singer proceed to compute ind $D_{X}$ for a general Dirac operator on a compact manifold with boundary. The problem splits into two pieces: a cylinder near $\partial X$ and $X \backslash \partial X$. On the cylinder they use the spectral decomposition (7.8) and the product metric decomposition (7.7) to convert $D_{X}$ to a family of ordinary differential operators parametrized by $\operatorname{spec}\left(A_{Y}\right)$. On the complement of $\partial X$ they use heat kernel methods, as in the local index theorem ( $₫ 6.2)$. Gluing the two regions via a partition of unity, they prove the following.

Theorem 7.9 (Atiyah, Patodi, and Singer APS1]). Let X be a compact Riemannian manifold with boundary, and assume the Riemannian metric is a product in a neighborhood of $\partial X$. Let $D_{X}$ be a generalized Dirac operator. Then with respect to the global boundary conditions,

$$
\text { ind } D_{X}=\int_{X} \omega-\frac{\eta_{\partial X}+h_{\partial X}}{2},
$$

where $\omega$ is the Chern-Weil form of the $\hat{A}$-genus, $\eta_{\partial X}$ is the $\eta$-invariant, and $h_{\partial X}=$ dim $\operatorname{ker} D_{\partial X}$.

To define the $\eta$-invariant on $Y=\partial X$, let $A_{Y}=\sigma^{-1} D_{Y}$ and form

$$
\eta(s)=\sum_{\lambda \in \operatorname{spec}\left(A_{Y}\right) \backslash\{0\}}(\operatorname{sign} \lambda)|\lambda|^{-s}, \quad \operatorname{Re}(s)>>0 .
$$

This is a Riemannian version of an $L$-function, an echo of the number-theoretic $L$-function in the signature defect on a Hilbert modular surface, and a variation on the Riemannian version (6.2) of a $\zeta$-function. The infinite sum in (17.11) converges for $\operatorname{Re}(s)>>0$, there is a meromorphic continuation 16 to the complex $s$-line, and $s=0$ is a regular point. Define $\eta_{Y}=\eta(0)$. We remark that the main theorem in [APS1] applies to more general first-order elliptic differential operators.

The index theorem, Theorem 7.9, simplifies for the signature operator (3.7). Let $X$ be a compact oriented Riemannian $4 k$-manifold with product metric near $\partial X$.

\footnotetext{
${ }^{16}$ Notably, $\eta(s)$ is holomorphic for $\operatorname{Re}(s)>-1 / 2$.
} 
The symmetric bilinear form (2.9) is nondegenerate restricted to the image of $H^{2 k}(X, \partial X ; \mathbb{R})$ in $H^{2 k}(X ; \mathbb{R})$, and $\operatorname{Sign}(X)$ is its signature.

Corollary 7.12 (Atiyah, Patodi, and Singer [APS1]). In this situation,

$$
\operatorname{Sign}(X)=\int_{X} \omega-\eta_{\partial X}
$$

where $\omega$ is the Chern-Weil form of the L-genus and $\eta_{\partial X}$ is the $\eta$-invariant of the self-adjoint operator on $\Omega^{\text {even }}(\partial X)$ given by $(-1)^{k+q+1}(* d-d *)$ on $\Omega^{q}(\partial X)$.

In particular, $-\eta$ is the signature defect (17.4). Note that the signature defect is a spectral invariant of a natural differential operator on $\partial X$, a property which is not apparent from its definition.

Remark 7.14. The signature defect also plays a star role in two of Atiyah's later papers [A8, A9].

The $\eta$-invariant is our first example of a geometric invariant of a Dirac operator. To illustrate, recall Example 5.13. Let $Y \rightarrow S$ be a proper fiber bundle of odd relative dimension equipped with a relative Riemannian spin structure. From this geometric data we obtain a family of self-adjoint Dirac operators parametrized by $S$. The lowest piece of the topological index is a homotopy class of maps $S \rightarrow \mathbb{R} / \mathbb{Z}$. The expression

$$
\xi_{Y / S}=\frac{\eta_{Y / S}+h_{Y / S}}{2}(\bmod 1): S \longrightarrow \mathbb{R} / \mathbb{Z}
$$

from (7.10) refines the homotopy class to a specific map, the geometric invariant in question. Also, Theorem 7.9 implies that the differential of (7.15) is

$$
d \xi_{Y / S}=\int_{Y / S} \omega
$$

where $\omega$ is the differential form in (7.10). This is a kind of "curvature" formula for the geometric invariant $\xi_{Y / S}$; there is an analogue for other geometric invariants of Dirac operators.

Atiyah, Patodi, and Singer [APS3, §7], in collaboration with Lusztig, gave another analytic computation of the homotopy class of the map $S \rightarrow \mathbb{R} / \mathbb{Z}$. For any loop $S^{1} \rightarrow S$ they prove the winding number of the composite $S^{1} \rightarrow S \rightarrow \mathbb{R} / \mathbb{Z}$ is the spectral flow of the pull-back family of Dirac operators parametrized by $S^{1}$. The spectral flow counts with sign the integer jumps in the $\xi$-invariant (7.15) as we travel around $S^{1}$. Alternatively, the union of the spectra of the Dirac operators is a closed subset $C \subset S^{1} \times \mathbb{R}$; the spectral flow is the intersection number of $C$ with $S^{1} \times\{0\}$.

Example 7.17. The simplest nontrivial spectral flow occurs for the family of complex self-adjoint Dirac operators

$$
D_{s}=\sqrt{-1} \frac{d}{d x}+s, \quad 0 \leq s \leq 1,
$$

acting on $\mathbb{R} / 2 \pi \mathbb{Z}$ with coordinate $x$. The operator $D_{1}$ is isomorphic to $D_{0}$ : conjugate by the multiplication operator $e^{\sqrt{-1} x}$. The union of spectra $C \subset S^{1} \times \mathbb{R}$ is a helix. 
7.4. Secondary geometric invariants. The most elementary secondary invariant is the total geodesic curvature of a curve in a Riemannian 2-manifold; it appears in the Gauss-Bonnet formula (7.2). The associated primary topological invariant is the Euler number. The generalizations below are more akin to the $\bmod \mathbb{Z}$ reduction of the total geodesic curvature, which is - up to a sign - the holonomy of the Levi-Civita connection.

Let $G$ be a Lie group with finitely many components, let $\pi: P \rightarrow X$ be a principal $G$-bundle, and let $\Theta \in \Omega^{1}(P ; \mathfrak{g})$ be a connection form. Let $p \in\left(\operatorname{Sym}^{k} \mathfrak{g}^{*}\right)^{G}$ be an Ad-invariant polynomial on the Lie algebra $\mathfrak{g}$. As we have already used, Chern-Weil associate to this data a closed differential form $p(\Omega) \in \Omega^{2 k}(X)$ that depends only on the curvature $\Omega$ of $\Theta$. Furthermore, it is natural in the connection $\Theta$. Its de Rham cohomology class $[p(\Omega)] \in H^{2 k}(X ; \mathbb{R})$ is independent of $\Theta$, so is an invariant of the principal bundle $\pi$. If $G$ is compact, which we now assume, then this invariant is derived from a characteristic class $c_{p} \in H^{2 k}(B G ; \mathbb{R})$ in the cohomology of the classifying space of $G$. In 1972 Chern and Simons CS introduced a secondary geometric invariant attached to a refinement of $c_{p}$ to an integral cohomology class $c \in H^{2 k}(B G ; \mathbb{Z})$. (Refinements exist only if the periods of $c_{p}$ are integers.) In this situation, the primary $\mathbb{Z}$-valued invariant is a characteristic number of a principal bundle $\pi: P \rightarrow X$ over a $2 k$-dimensional closed oriented manifold. The secondary $\mathbb{R} / \mathbb{Z}$-valued Chern-Simons invariant is defined for $\pi: Q \rightarrow Y$ with connection $\Theta$, where $Y$ is a closed oriented $(2 k-1)$-dimensional manifold. The secondary $\mathbb{R} / \mathbb{Z}$ valued invariant depends on the connection, whereas the primary $\mathbb{Z}$-valued invariant is topological.

Example 7.19. For $N \in \mathbb{Z}^{\geq 3}$ let $p_{1} \in H^{4}\left(B \mathrm{SO}_{N} ; \mathbb{Z}\right)$ be the universal first Pontrjagin class of a principal $\mathrm{SO}_{N}$-bundle. Working intrinsically - that is, with the tangential geometry of manifolds - the $\mathbb{Z}$-valued primary invariant of a closed oriented 4-manifold $X$ is

$$
p_{1}(W)[W] .
$$

The secondary invariant $\Gamma(Y)$ on a closed oriented Riemannian 3-manifold $Y$ is the Chern-Simons invariant of its Levi-Civita connection. In this case [CS, $\S 6], \Gamma(Y)$ is a conformal invariant and an obstruction to the existence of a conformal immersion $Y \rightarrow \mathbb{E}^{4}$, where $\mathbb{E}^{4}$ is Euclidean 4 -space.

Remark 7.21. The Chern-Simons invariant finds a natural expression in differential cohomology [ChS, $\mathrm{F} 2, \mathrm{HS}$, which unifies the primary and secondary invariants in a single framework.

The Atiyah-Patodi-Singer $\eta$-invariant is a secondary invariant analogous to the Chern-Simons invariant, but in index theory rather than the theory of characteristic classes. Let $Y$ be a spin Riemannian manifold, and let $\pi: Q \rightarrow Y$ be a principal $G$-bundle with connection. Whereas the Chern-Simons story begins with an integral cohomology class $c \in H^{\bullet}(B G ; \mathbb{Z})$, to define the $\eta$-invariant, we begin with a complex 17 linear representation $\rho: G \rightarrow \operatorname{Aut}(V)$; the isomorphism class of $\rho$ is an element of the equivariant $K$-theory group $K_{G}^{0}(\mathrm{pt})$. From the beginning we see the Chern-Simons invariant pertains to integer cohomology, while the $\eta$-invariant pertains to $K$-theory. Form the Dirac operator on $Y$ coupled to the associated vector bundle $V_{Q} \rightarrow Y$ with its inherited covariant derivative. Then the $\mathbb{R} / \mathbb{Z}$-valued

\footnotetext{
${ }^{17}$ There is a refinement to real representations and real $K O$-theory.
} 
invariant

$$
\xi_{Y}(V)=\frac{\eta_{Y}(V)+h_{Y}(V)}{2}(\bmod 1)
$$

depends smoothly on the Riemannian metric and the connection on $\pi 18$ The corresponding primary invariant is the $\mathbb{Z}$-valued index of the twisted Dirac operator on manifolds of dimension $\operatorname{dim} Y+1$ equipped with a principal $G$-bundle.

Remark 7.23. To obtain a nontopological invariant, we must have $\operatorname{dim} Y$ odd. The $\eta$-invariant also leads to new and interesting topological invariants in even dimensions, for example on unoriented manifolds with a pin structure [Gi2].

Example 7.24. Consider $\operatorname{dim} Y=3$ as in Example [7.19 but now assume $Y$ is a closed spin Riemannian manifold. The expression (7.22) for the standard Dirac operator (no principal $G$-bundle) is the $\mathbb{R} / \mathbb{Z}$-valued secondary invariant of the index of the Dirac operator on a closed spin 4 -manifold $X$, which by the index theorem (4.7) is

$$
\text { ind } D_{X}=-\frac{1}{24} p_{1}(T W)[W] .
$$

The difference with (7.20) is the rational factor. The integrality of the $\hat{A}$-genus (recall (2.26) ) implies (7.25) is an integer. Turning to the secondary invariants $\Gamma(Y)$ and $\xi_{Y}$, since they are $\mathbb{R} / \mathbb{Z}$-valued, we cannot multiply $\Gamma(Y)$ by a nonintegral rational number like $-1 / 24$; instead we clear denominators and compare $-24 \xi_{Y}$ with $\Gamma(Y)$. In the case at hand they agree; a similar comparison in more general circumstances leads to a spin bordism invariant. In any case, we see that the $\eta$-invariant is a more subtle invariant than the Chern-Simons invariant. Put differently, the secondary invariants based on $K$-theory contain refined information over those based on integer cohomology. This echoes the stronger topological information derived from integrality of the primary invariants; see 2.4 .

Remark 7.26.

(1) As a concrete illustration of this extra power, Atiyah, Patodi, and Singer APS2, §4] use the $\eta$-invariant of the signature operator to refine the ChernSimons obstruction to conformal embeddings $Y^{3} \rightarrow \mathbb{E}^{4}$. Also, they show how to use $\eta$-invariants to construct the Adams $e$-invariant, an invariant of framed bordism.

(2) The primary indices and secondary $\eta$-invariants are unified in the framework of differential $K$-theory; compare Remark $[7.21$, See $[\mathrm{FL}]$ and the references therein.

(3) The Atiyah-Patodi-Singer $\eta$-invariant appears in many contexts in geometry and beyond. It also, together with other characters in topological and geometric index theory, makes many appearances in theoretical physics: quantum field theory, string theory, and condensed matter theory.

7.5. Determinants of Dirac operators. Before proceeding to Dirac operators, consider a second-order Laplace operator $\Delta$ on a closed manifold $X$, as in 6.1 .

\footnotetext{
${ }^{18}$ For the special case of the signature operator, in which the kernel — the harmonic forms - have cohomological significance, the $\mathbb{R}$-valued invariant is smooth. (It appears in (7.13).)
} 
Then $\Delta$ has a discrete spectrum consisting of eigenvalues $0 \leq \lambda_{1} \leq \lambda_{2} \leq \cdots$, repeated with multiplicity. Formally, the determinant of $\Delta$ is

$$
\operatorname{det} \Delta "=" \prod_{m=1}^{\infty} \lambda_{m} .
$$

(Assume $\lambda_{1}>0$ or omit the zero eigenvalues to avoid $\operatorname{det} \Delta=0$.) Of course, this infinite product diverges. For example, if $X=S^{1}$ and $\Delta$ is the usual scalar Laplace operator, then up to a constant the infinite product is $\prod_{m=1}^{\infty} m^{2}$ after omitting the zero eigenvalue. One way to impart a value to this infinite product, pioneered by Ray and Singer RS in 1971 and following a technique familiar in complex analysis $\mathrm{JL}$, is to use the analytic continuation of the $\zeta$-function (6.2), which is defined as

$$
\zeta_{\Delta}(s)=\sum_{m=1}^{\infty} \lambda^{-s}, \quad s \in \mathbb{C},
$$

for $\operatorname{Re}(s)>>$. Then in the region of absolute convergence of the infinite sum, we differentiate

$$
-\zeta_{\Delta}^{\prime}(s)=\sum_{m=1}^{\infty} \lambda^{-s} \log \lambda,
$$

and then use the regularity of the analytic continuation of $\zeta_{\Delta}$ at $s=0$ to define

$$
\operatorname{det} \Delta:=e^{-\zeta_{\Delta}^{\prime}(0)} \text {. }
$$

For the scalar Laplace operator on $S^{1}$, the elliptic $\zeta$-function defined in (7.28) reduces to the Riemann $\zeta$-function, up to a constant.

The first-order Dirac operator (4.6) is not self-adjoint; its domain and codomain are different. A finite-dimensional model is a linear operator

$$
T: V^{0} \longrightarrow V^{1}
$$

between different vector spaces $V^{0}, V^{1}$. There is an induced map $\bigwedge^{q} T: \bigwedge^{q} V^{0} \rightarrow$ $\bigwedge^{q} V^{1}$ on each exterior power. If $\operatorname{dim} V^{0}=\operatorname{dim} V^{1}$, then the induced map for $q=\operatorname{dim} V^{i}$ is the determinant

$$
\operatorname{det} T: \operatorname{Det} V^{0} \longrightarrow \operatorname{Det} V^{1},
$$

where Det $V^{i}=\bigwedge^{\operatorname{dim} V^{i}}\left(V^{i}\right)$ is the determinant line. If $V^{0}=V^{1}$, then the operator $\operatorname{det} T$ is multiplication by the numerical determinant. But in genera $19 \operatorname{det} T$ is an element of a line, namely the one-dimensional vector space

$$
\operatorname{Hom}\left(\operatorname{Det} V^{0}, \operatorname{Det} V^{1}\right)
$$

called the determinant line. The determinant construction generalizes to Fredholm operators (7.31), where now $V^{0}, V^{1}$ are typically infinite dimensional. The formula (7.33) does not make sense if $V^{0}, V^{1}$ are infinite dimensional; rather, the determinant line is defined using the finite dimensionality of the kernel and cokernel. Quillen Q constructs a determinant line bundle $\pi$ : Det $\rightarrow$ Fred over the space of Fredholm operators together with a continuous section det: Fred $\rightarrow$ Det. It is the next topological invariant of Fredholm operators after the numerical index (3.13).

\footnotetext{
${ }^{19}$ If $\operatorname{dim} V^{0} \neq \operatorname{dim} V^{1}$, define $\operatorname{det} T=0$.
} 
Let $f: X \rightarrow S$ be a proper fiber bundle with fibers of even-dimension $n$, and assume $f$ is endowed with a relative Riemannian spin structure, as in Example 5.13. For a complex vector bundle $V \rightarrow X$ with covariant derivative, the index of the resulting family $D_{X / S}(V)$ of complex Clifford linear Dirac operators is computed by the pushforward

$$
f_{!}: K^{0}(X) \longrightarrow K^{-n}(S)
$$

The numerical index is the image of $f_{!}[V]$ under $K^{-n}(S) \rightarrow H^{0}(S ; \mathbb{Z})$. Parallel to (5.15), the next lowest piece of the topological index is computed by a natural map

$$
\operatorname{Det}: K^{-n}(S) \longrightarrow H^{2}(S ; \mathbb{Z}) \text {. }
$$

The index theorem for families implies that the topological equivalence class of the Fredholm determinant line bundle

$$
\operatorname{Det} D_{X / S}(V) \longrightarrow S
$$

is Det $f_{!}[V] \in H^{2}(S ; \mathbb{Z})$.

There is a geometric refinement of this topological piece of the index. Its analytic expression is a hermitian connection and compatible covariant derivative on the determinant line bundle (7.36). The Quillen metric $\mathrm{Q}$ is constructed using the $\zeta$-function determinant (7.30), and the covariant derivative uses a similar-but somewhat more subtle- $\zeta$-function BiF1. The isomorphism class of a line bundle with covariant derivative is determined by its holonomy around loops. Base change along a loop $\varphi: S^{1} \rightarrow S$ gives rise to a fiber bundle $X_{\varphi} \rightarrow S^{1}$ and vector bundle $V_{\varphi} \rightarrow X_{\varphi}$. The bounding spin structure on $S^{1}$ combines with the relative spin structure on $X_{\varphi} / S^{1}$ to produce a spin structure on $X_{\varphi}$. Choose an arbitrary metric $g_{S^{1}}$ on $S^{1}$, and let $X_{\varphi}(\epsilon)$ be the manifold $X_{\varphi}$ with Riemannian metric $g_{S^{1}} / \epsilon^{2} \oplus g_{X_{\varphi} / S^{1}}$. (The direct sum is with respect to the horizontal distribution in the relative Riemannian structure.) Then the holonomy around the loop $\varphi$ is [BiF2]

$$
\operatorname{hol}_{\varphi} \operatorname{Det} D_{X / S}(V)=\lim _{\epsilon \rightarrow 0} e^{-2 \pi i \xi_{X \varphi}(\epsilon)}(V),
$$

where the exponent is the Atiyah-Patodi-Singer $\eta$-invariant (17.22). This holonomy formula was inspired by Witten's global anomaly Wi3. The curvature of the determinant line bundle is

$$
\operatorname{curv} \operatorname{Det} D_{X / S}(V)=2 \pi i \int_{X / S} \omega
$$

where $\omega$ is the Chern-Weil form that represents $\hat{A}(X / S) \smile \operatorname{ch}(V)$; compare (7.16). 
Remark 7.39.

(1) The adiabatic limit in (7.37) was introduced in Wi3. Other geometric interpretations of Witten's global anomaly formula were given in [Che, S2].

(2) The holonomy formula (7.37) may be regarded as a Fubini theorem relating the geometric invariants $\eta$ and Det, once one knows that the holonomy of a line bundle $L \rightarrow S^{1}$ is the exponentiated $\eta$-invariant of $D_{S^{1}}(L)$.

(3) As in Remark 7.26(2), the isomorphism class of the determinant line bundle with its metric and covariant derivative can be computed by a pushforward in differential $K$-theory. This refines the topological index theorem which computes the topological isomorphism class of (7.36) as Det $f_{!}[V]$.

\section{AnOmalies AND INDEX THEORY}

In part inspired by Is Singer's advocacy of theoretical physics as a fertile ground for geometers, beginning in the late 1970s Michael Atiyah turned his attention to geometric problems in quantum field theory and, later, string theory. Simon Donaldson's paper [Do in this volume covers the burst of activity in the late 1970s and early 1980s emanating from the Yang-Mills equations. The Atiyah-Singer index theorem is a part of that story, but I will restrict my exposition here to Atiyah's work in the late 1980s on anomalies and on his axiomatization of topological field theory.

We begin in 8.1 by arguing that anomalies are an expression of the projective (as opposed to linear) nature of quantum theory. The geometrical and topological link between anomalies and index theory was initiated in a joint paper of Atiyah and Singer, which we summarize in 98.2 . Atiyah's axioms for topological field theory, and their relationship to bordism in algebraic topology, are the subjects of 8.3 . We conclude in 8.4 by mentioning the modern point of view on anomalies, which ties together the two aforementioned Atiyah works. The specialization to spinor fields brings in topological and geometric index theorems as well, all synthesized in a general anomaly formula which draws on many of the Atiyah papers we have discussed.

8.1. Projectivity and symmetries in quantum mechanics. Anomalies are often said to be the failure of a classical symmetry to hold in a corresponding quantum system. More precisely, a quantum symmetry is projective and the anomaly is the obstruction to linearization. Quantum theory is inherently projective, and the anomaly - in a more general sense than a notion tied to symmetry-encodes the projectivity of a quantum system.

A quantum mechanical system is specified 20 by a triple of data $(\mathscr{P}, p, H)$. The space $\mathscr{P}$ is a projective space, the projectivization $\mathbb{P} \mathscr{H}$ of a complex separable Hilbert space $\mathscr{H}$, but $\mathscr{H}$ is not singled out. One way to define $\mathscr{P}$ is to fix $\mathscr{H}_{0}$ and let $\mathscr{C}_{\mathscr{P}}$ be the groupoid whose objects are pairs $(\mathscr{H}, \theta)$ of a Hilbert space and a projective linear isomorphism $\theta: \mathbb{P} \mathscr{H}_{0} \rightarrow \mathbb{P} \mathscr{H}$. Morphisms $(\mathscr{H}, \theta) \rightarrow\left(\mathscr{H}^{\prime}, \theta^{\prime}\right)$ are linear isometries $\mathscr{H} \rightarrow \mathscr{H}^{\prime}$ whose projectivization commutes with $\theta, \theta^{\prime}$; they form a torsor over the unitary group $\mathbb{T} \subset \mathbb{C}^{\times}$of unit norm scalars. The basepoint $\mathscr{H}_{0}$ is not part of the structure. Define $\mathscr{P}$ as the limit of $\mathbb{P} \mathscr{H}$ over all $(\mathscr{H}, \theta) \in \mathscr{C}_{\mathscr{P}}$.

\footnotetext{
${ }^{20}$ There is a more general framework for quantum theory using $C^{*}$-algebras, but for this exposition the simpler context suffices.
} 
Also, define the $*$-algebra $\mathfrak{A}_{\mathscr{P}}$ (of observables) as the limit of End $\mathscr{H}$ over $(\mathscr{H}, \theta) \in$ $\mathscr{C}_{\mathscr{P}}$. (End $\mathscr{H}$ is the algebra of bounded linear operators on $\mathscr{H}$; for simplicity, we omit unbounded operators from this exposition.) The projective space $\mathscr{P}$ is the space of pure states of the quantum system. Embed $\mathscr{P} \hookrightarrow \mathfrak{A}_{\mathscr{P}}$ as rank 1 orthogonal projections. Then mixed states are convex combinations of pure states. The function

$$
\begin{aligned}
p: \mathscr{P} \times \mathscr{P} & \longrightarrow[0,1] \\
L_{1}, L_{2} & \longmapsto\left|\left\langle\psi_{1}, \psi_{2}\right\rangle\right|^{2}, \quad \psi_{i} \in L_{i},
\end{aligned}
$$

is used to compute transition probabilities. To define $p$, choose $(\mathscr{H}, \theta) \in \mathscr{C}_{\mathscr{P}}$ and identify $\mathscr{P}$ with $\mathbb{P} \mathscr{H}$; then $L_{i} \subset \mathscr{H}$ is a line and $\psi_{i} \in L_{i}$ is a unit norm vector. The Hamiltonian $H$ is a self-adjoint element of $\mathfrak{A}_{\mathscr{P}}$, usually assumed to have spectrum bounded below.

Fix $(\mathscr{H}, \theta) \in \mathscr{C}_{\mathscr{P}}$. Let $G(\mathscr{H})$ be the infinite-dimensional Lie group with identity component the group $U(\mathscr{H})$ of unitary automorphisms of $\mathscr{H}$ and off-identity component the torsor of antiunitary automorphisms. A basic theorem of Wigner asserts that the sequence of Lie group homomorphisms

$$
1 \longrightarrow \mathbb{T} \longrightarrow G(\mathscr{H}) \stackrel{q}{\longrightarrow} \operatorname{Aut}(\mathbb{P} \mathscr{H}, p) \longrightarrow 1
$$

is a group extension: $q$ is surjective. Let $G$ be a Lie group of symmetries of $(\mathscr{P}, p)$, i.e., a homomorphism $G \rightarrow \operatorname{Aut}(\mathscr{P}, p)$. By pullback, we obtain a group extension

$$
1 \longrightarrow \mathbb{T} \longrightarrow G_{\mathscr{H}} \longrightarrow G \longrightarrow 1
$$

and a $\mathbb{Z} / 2 \mathbb{Z}$-grading $\epsilon: G \rightarrow \mathbb{Z} / 2 \mathbb{Z}$. The $\mathbb{Z} / 2 \mathbb{Z}$-grading is independent of $(\mathscr{H}, \theta)$, as is the isomorphism class of the extension (8.3). The $\mathbb{Z} / 2 \mathbb{Z}$-graded group extension (8.3) can be called the anomaly; it measures the projectivity of the symmetry.

\section{Remark 8.4.}

(1) If $(\mathscr{P}, p, H)$ has a classical limit and the symmetry persists in the limit, then it is the Lie group $G$ which acts on the classical system. The passage from $G$ to $G_{\mathscr{H}}$ is what was referenced in the first paragraph of this section.

(2) Suppose we have a family of quantum systems with parameter manifold $S$, so in particular a fiber bundle $\mathscr{P} \rightarrow S$ of projective Hilbert spaces. In some physical situations one wants to "integrate over $S$ " to form a new quantum mechanical system. If we write $\mathscr{P} \rightarrow S$ as the projectivization of a vector bundle $\mathscr{E} \rightarrow S$ of Hilbert spaces, then the state space of the integrated system is the space of $L^{2}$ sections of $\mathscr{E} \rightarrow S$, assuming a measure on $S$. The anomaly is the obstruction to lifting $\mathscr{P} \rightarrow S$ to a vector bundle, a necessary first step to integrate out $S$. On the other hand, in the absence of integrating out $S$, the anomaly is not an obstruction but rather a feature of a family of quantum systems, useful in many contexts.

(3) The isomorphism class of the central extension (8.3) lives in the cohomology group $H^{2}(G ; \mathbb{T})$. (The type of cohomology depends on the type of group. Nothing is lost here by assuming that $G$ is a finite group.) If we drop a cohomological degree, then $H^{1}(G ; \mathbb{T})$ is the group of one-dimensional unitary representations. Therefore, the projectivity measured in 8.3 is 
obtained via a 2 -step procedure starting with linear actions of $G$ on $\mathscr{H}$ :

(i) replace $\mathscr{H}$ by a one-dimensional vector space, and

(ii) interpret one-dimensional representations of $G$ cohomologically and raise the cohomological degree by 1 .

We will see an analogous procedure in quantum field (88.4).

Let $t_{0}<t_{1}<\cdots<t_{n+1}$ be real numbers, thought of as points on the affine time line, and let $A_{1}, \ldots, A_{n} \in \mathfrak{A}_{\mathscr{P}}$. Fix initial and final pure states $L_{0}, L_{n+1} \in \mathscr{P}$. A basic quantity of interest in quantum mechanics is the probability ${ }^{21}$

$$
p\left(L_{n+1}, e^{-i\left(t_{n+1}-t_{n}\right) H / \hbar} A_{n} \cdots e^{-i\left(t_{2}-t_{1}\right) H / \hbar} A_{1} e^{-i\left(t_{1}-t_{0}\right) H / \hbar} L_{0}\right),
$$

where $\hbar$ is Planck's constant. Physical questions may be phrased in terms of these probabilities. Fix $(\mathscr{H}, \theta) \in \mathscr{C}_{\mathscr{P}}$, identify $\mathscr{P} \approx \mathbb{P} \mathscr{H}$, and choose unit norm vectors $\psi_{0} \in L_{0}$ and $\psi_{n+1} \in L_{n+1}$. The amplitude, or correlation function, of this data is the complex number

$$
\left\langle\psi_{n+1}, e^{-i\left(t_{n+1}-t_{n}\right) H / \hbar} A_{n} \cdots e^{-i\left(t_{2}-t_{1}\right) H / \hbar} A_{1} e^{-i\left(t_{1}-t_{0}\right) H / \hbar} \psi_{0}\right\rangle_{\mathscr{H}} .
$$

More invariantly, the data $L_{0}, L_{n+1}, t_{0}, \ldots, t_{n+1}, A_{1}, \ldots, A_{n}$ determine a hermitian line $\mathcal{L}$, and the amplitude is an element of $\mathcal{L}$ whose norm is the probability (8.5).

Remark 8.7. The fact that the amplitudes of $(\mathscr{P}, p, H)$ lie in a line $\mathcal{L}$ without a distinguished basis element is another aspect of the projectivity of quantum mechanics. The lines $\mathcal{L}$ form a line bundle over a parameter space of data. These line bundles are part of the anomaly of the quantum mechanical system, a counterpart for correlation functions of the projective bundles in Remark 8.4(2).

The context for anomalies sketched in this section is soft, much as is index theory for general Fredholm operators. Quantum field theory brings in the geometry of Wick-rotated spacetimes, and in that context anomalies also exhibit more geometry, much as does index theory for Dirac operators. This is more than an analogy in the case of fermionic fields, to which we now turn.

8.2. Spinor fields and anomalies in quantum field theory. Geometric links between anomalies for spinor fields and index theory were forged in a 1984 paper of Atiyah and Singer AS7. (Other contemporaneous papers, such as AgW, AgG,Lt2] also brought index polynomials into the theory of anomalies.) The setup is gauge theory in physics. (By that time Atiyah had already achieved many results in mathematical gauge theory; see the article [Do] by Donaldson in this volume.) We first summarize their work and then relate it to the geometric picture of anomalies.

Let $n$ be an even positive integer, and let $X$ be a closed Riemannian spin manifold of dimension $n$. Suppose $P \rightarrow X$ is a principal bundle with structure group a compact Lie group $G$. Let $\mathcal{A}$ be the infinite-dimensional affine space of connections on $P \rightarrow X$, and let $\mathscr{G}$ be the group of gauge transformations: automorphisms of $P \rightarrow X$ which act as the identity on $X$. Then $\mathscr{G}$ acts on $\mathcal{A}$. Assume $X$ is connected, fix a basepoint $p_{0} \in P$, and let $\mathscr{G}_{0} \subset \mathscr{G}$ be the subgroup of gauge transformations which fix $p_{0}$. Then $\mathscr{G}_{0}$ acts freely on $\mathcal{A}$, and in the sequence

$$
\frac{\mathcal{A} \times P}{\mathscr{G}_{0}} \stackrel{\pi}{\longrightarrow} \frac{\mathcal{A}}{\mathscr{G}_{0}} \times X \stackrel{\mathrm{pr}_{1}}{\longrightarrow} \frac{\mathcal{A}}{\mathscr{G}_{0}},
$$

\footnotetext{
${ }^{21}$ If the linear operator $e^{-i\left(t_{n+1}-t_{n}\right) H / \hbar} A_{n} \cdots e^{-i\left(t_{2}-t_{1}\right) H / \hbar} A_{1} e^{-i\left(t_{1}-t_{0}\right) H / \hbar}$ is zero on $L_{0}$, the probability is zero.
} 
$\pi$ is a principal $G$-bundle and $\operatorname{pr}_{1}$ is a product fiber bundle with fiber $X$. To a unitary representation of $G$, we associate a vector bundle to $\pi$. This data determines a topological index

$$
\text { ind } \in K^{-2 n}\left(\mathcal{A} / \mathscr{G}_{0}\right) \cong K^{0}\left(\mathcal{A} / \mathscr{G}_{0}\right) \text {. }
$$

Atiyah and Singer construct closed differential forms which represent $\operatorname{ch}($ ind $) \in$ $H^{\bullet}\left(\mathcal{A} / \mathscr{G}_{0} ; \mathbb{R}\right)$ as follows. Fix a bi-invariant Riemannian metric on $G$. Then for each connection $A \in \mathcal{A}$, the manifold $P$ has a Riemannian metric which makes the projection $P \rightarrow X$ a Riemannian submersion in which the horizontal subspaces of the connection are orthogonal to the fibers. Use the $L^{2}$ metric on $\mathcal{A}$ to form a warped product metric on $\mathcal{A} \times P$. Then $\mathscr{G}_{0} \times G$ acts by isometries, and a connection on $\pi$ results by taking orthogonals to the $G$-orbits on the quotient by $\mathscr{G}_{0}$. The associated vector bundle inherits a covariant derivative, so there is a family of Dirac operators on $X$ parametrized by $\mathcal{A} / \mathscr{G}_{0}$, and the index theorem for families (\$5.5) implies that ind in (8.9) equals its analytic index. The Chern-Weil procedure produces the desired differential forms from the differential geometric data. In particular, $\operatorname{ch}_{1}($ ind $) \in H^{2}\left(\mathcal{A} / \mathscr{G}_{0} ; \mathbb{R}\right)$ is represented by

$$
\int_{X} \omega
$$

for a $(2 n+2)$-form $\omega$ on $\left(\mathcal{A} / \mathscr{G}_{0}\right) \times X$. Atiyah and Singer transgress $\omega$ to a $(2 n+1)$ form on $\mathscr{G}_{0} \times X$, and it is this differential form which appears in the contemporaneous physics literature as the anomaly of a spinor field in quantum field theory. They also relate their result to determinants. In particular, they interpret the cohomology class $c_{1}$ (ind) $\in H^{2}\left(\mathcal{A} / \mathscr{G}_{0} ; \mathbb{Z}\right)$ of $(8.10)$ as the isomorphism class of the determinant line bundle of the family of Dirac operators on $X$ parametrized by $\mathcal{A} / \mathscr{G}_{0}$.

The determinant, or more generally pfaffian, of a Dirac operator arises directly in Wick-rotated quantum field theories with spinor fields, as we now sketch in a general context. Let $n$ be a positive integer, let $G$ be a compact Lie group, and let $\rho$ be a representation of $G$. Suppose

$$
P \stackrel{\pi}{\longrightarrow} X \stackrel{f}{\longrightarrow} S
$$

is a principal $G$-bundle $\pi$ with connection and a proper fiber bundle $f$ of relative dimension $n$, the latter equipped with a relative Riemannian spin structure. As in 5.5. this data produces a family of Dirac operators $D_{X / S}$ parametrized by $S$. The Feynman integral over the spinor field $\psi$ is an infinite-dimensional variant of a standard Gaussian integral:

$$
\int_{\mathscr{S}_{s}} e^{-\left(\psi, D_{s} \psi\right)}
$$

For each $s \in S$ we are meant to integrate over the infinite-dimensional vector space $\mathscr{S}_{s}$ of spinor fields. The Dirac operator $D_{s}$ appears in the exponent, which is a skew-symmetric bilinear form on $\mathscr{S}_{s} 22$ By analogy with finite-dimensional integrals, 8.12) is defined to be pfaff $D_{s}$, the pfaffian of the Dirac operator. In special cases, such as the Atiyah-Singer setup above, the pfaffian reduces to a determinant,

\footnotetext{
${ }^{22}$ Physicists begin with a relativistic theory on Minkowski spacetime in Lorentz signature, and then they Wick-rotate to Riemannian manifolds. What emerges naturally is the bilinear form in the exponent.
} 
and we make that simplification here. Then the result of the integral (8.12) is the section det $D_{X / S}$ of the determinant line bundle

$\operatorname{Det} D_{X / S} \longrightarrow S$

as in $\$ 7.5$

Remark 8.14

(1) The integral (8.12) is an example of a correlation function in Feynman's approach to quantum field theory. The fact that it is an element of a complex line, rather than a complex number, is precisely the situation of anomalous amplitudes discussed in 88.1 . Here the determinant line bundle (8.13), including its metric and covariant derivative, is the anomaly.

(2) The universal parameter space $S$, for a fixed manifold $X$, is the space of Riemannian metrics and $G$-connections on $X$. In a quantum field theory one often wants to integrate over the metric or connections or both. The anomaly is an obstruction to doing so.

The anomaly is the obstruction to a trivialization of 8.13). The topological first Chern class of the index discussed by Atiyah and Singer (cf. [S3]) obstructs a nonzero section, but one needs something sharper. In F3 the anomaly is identified as the obstruction to a flat section, relative to the natural connection BiF1. This leads to an interpretation of the 2-form (8.10) computed by Atiyah and Singer as the curvature of this natural connection. Physicists call this local obstruction to a flat section the local anomaly; the holonomy is the global anomaly of Witten Wi3. Indeed, the developments in geometric index theory recounted in $\$ 7.5$ were directly inspired by this geometry of anomalies.

Remark 8.15. The anomaly as an obstruction to lifting a bundle of projective spaces to a vector bundle (Remark 8.4(2)) is measured by the next invariant in geometric index theory after the determinant line bundle - the Dirac gerbe $\mathrm{Lt1}, \mathrm{Bu}$. We refer the reader to $\mathrm{FS}$, Seg2, NAg.

8.3. Topological field theory and bordism. Atiyah's engagement with quantum field theory went well beyond the index theorem. One particularly influential paper A11] sets out axioms for topological quantum field theories, parallel to axioms introduced previously by Segal [Seg3 for two-dimensional conformal field theories. One key impetus was Witten's quantum Chern-Simons theory Wi4, which places the Jones invariants of knots in a manifestly three-dimensional framework. But there were many other examples too that Atiyah abstracted into his axioms. His paper A11 is dedicated to Thom, and indeed bordism theory is very much at the forefront. Atiyah [A12] writes the following about this paper:

Because mathematicians are frightened by the Feynman integral and are unfamiliar with all the jargon of physicists, there seemed to me to be a need to explain to mathematicians what a topological quantum field theory really was, in user-friendly terms. I gave a simple axiomatic treatment (something mathematicians love) and listed the examples that arise from physics. The task of the mathematician is then to construct, by any method possible, a theory that fits the axioms. I like to think of this as analogous to the Eilenberg-Steenrod axioms of cohomology, where one can use simplicial, Cech or de Rham methods to construct the theory. This 
last is closest to physics but the others have some advantages. In the quantum field theory context, where things are vastly more difficult, the combinatorial approach is so far the only one that has been made to work (for the Jones polynomials).

Here we give a concise version of the axioms.

As motivation, recall the signature of a closed oriented manifold of dimension $4 k$ for some $k \in \mathbb{Z}^{\geq 0}$. As used crucially in the proof of Theorem 2.11, the signature is a bordism invariant, that is, a homomorphism of abelian groups

$$
\text { Sign : } \Omega_{4 k}(\mathrm{SO}) \longrightarrow \mathbb{Z},
$$

where $\Omega_{4 k}(\mathrm{SO})$ is Thom's bordism group of closed oriented $4 k$-manifolds. A topological field theory is a "categorified bordism invariant". Fix a nonnegative integer $d$. (The relation to $n$ in $\$ 8.2$ is $n=d+1$.) Let $\operatorname{Bord}_{\langle d, d+1\rangle}$ be the following category, first introduced by Milnor Mi2]. The objects are closed $d$-manifolds. If $Y_{0}, Y_{1}$ are two such, then a morphism $Y_{0} \rightarrow Y_{1}$ is represented by a compact $(d+1)$ manifold $X$ with boundary partitioned as $\partial X=Y_{0} \amalg Y_{1}$. In other words, $X$ is a bordism from $Y_{0}$ to $Y_{1}$. Diffeomorphic bordisms rel boundary represent the same morphism. Composition glues bordisms and disjoint union of manifolds provides a symmetric monoidal structure.

Remark 8.17.

(1) If we declare objects $Y_{0}, Y_{1}$ of $\operatorname{Bord}_{\langle d, d+1\rangle}$ to be equivalent if there exists a morphism $Y_{0} \rightarrow Y_{1}$, then the set of equivalence classes is the bordism group $\Omega_{d}$. In this sense, $\operatorname{Bord}_{\langle d, d+1\rangle}$ "categorifies" $\Omega_{d}$.

(2) A small variation yields bordism categories with tangential structure, such as an orientation.

We can now state the axioms. Let $k$ be a field, and let Vect $k$ be the category of $k$-vector spaces and linear maps. (In quantum theories, $k=\mathbb{C}$.) The operation of tensor product defines a symmetric monoidal structure on Vect $_{k}$.

Definition 8.18 (Atiyah A11]). A topological field theory is a symmetric monoidal functor

$$
F: \operatorname{Bord}_{\langle d, d+1\rangle} \longrightarrow \operatorname{Vect}_{k} .
$$

This definition is sometimes referred to as the Atiyah-Segal axiom system, and with suitable modifications and extensions it is believed to apply widely to field theories in mathematics and physics. The viewpoint in these axioms is very different from what one sees in physics texts. The Atiyah-Segal axiom system has provided a generation of mathematicians with a point of entry to this physics, they are the structure upon which many mathematical developments have been built, and they have illuminated geometric aspects of quantum field theories in physics as well.

To illustrate Definition 8.18 , we show how to extract numerical invariants of a normally framed knot $K \subset M$ in a closed 3-manifold $M$ from a field theory $F$ with $d=2$. Let $X$ be the 3 -manifold obtained from $M$ by removing an open tubular neighborhood of $K$. The result is a bordism $X: \partial X \rightarrow \emptyset^{2}$ from $\partial X$ to the empty 2-manifold. A normal framing of $K$ provides an isotopy class of diffeomorphisms $\partial X \approx S^{1} \times S^{1}$. Hence the value of the field theory $F$ on $X$ is a linear map

$$
F(X): V \longrightarrow k,
$$


where $V=F\left(S^{1} \times S^{1}\right)$ is the vector space attached to the standard 2-torus. For each vector $\xi \in V$, which may be viewed as a "label" attached to $K$, we obtain a numerical invariant $F(X)(\xi)$. The Jones invariants of knots are of this type. This is one of the key observations in Wi4.

8.4. Synthesis. We conclude by bringing together the Atiyah-Singer work on anomalies ( 8.2 ), the Atiyah-Segal axiom system for quantum field theory ( 8.3$)$, and the index theorems in $\S \& 4,5,7$,

One starting point is Remark 8.14 (2), which tells that an anomaly must be trivialized to construct a quantum field theory by integrating over certain fields, such as metrics or connections. (Such integrals are problematic analytically, but the anomaly and trivializations are mathematically well-defined.) Now if the resulting quantum field theory is to be local - and locality is a characteristic feature of quantum field theories - then the trivializations of the anomaly must be coherent in the background data (8.11). That coherence is precisely what is expressed in the Atiyah-Segal axiom system and its extensions. This is one line of reasoning which leads to the realization that an anomaly itself is a quantum field theory 23 albeit of a very special type. For the spinor field in 8.2 , the determinant lines in the fibers of (8.13) are one-dimensional state spaces in an $(n+1)$-dimensional field theory. This anomaly theory is invertible, but is not necessarily topological. (An invertible field theory (8.19) factors through the subgroupoid Line ${ }_{k} \subset$ Vect $_{k}$ of lines and invertible linear maps.)

Remark 8.21. We arrive at the same picture by following the ideas of 88.1 . Namely, a field theory in the form (8.19) is a linear representation of bordism, but quantum theory is projective and the anomaly measures the projectivity. Furthermore, Remark 8.4(3) gives a roadmap to locate this measurement. Here the bordism category plays the role of the group $G$. First, replace Vect ${ }_{k}$ by the category of onedimensional vector spaces and invertible linear maps. What results is an invertible field theory. Second, we interpret an invertible field theory cohomologically and raise the cohomological degree by 1 . The cohomological interpretation was introduced in [FHT; see [FH, §5]. We arrive at the same conclusion: the anomaly, or measurement of projectivity, of a field theory is an invertible $(n+1)$-dimensional field theory 24

This already brings together the aforementioned 1980s work of Atiyah, but we can go much further. Whereas a general field theory is a functor between symmetric monoidal categories, an invertible field theory can be formulated in stable homotopy theory, as a map of spectra. The domain, rather than a bordism category, is a bordism spectrum of the type 25 introduced by Thom. (We remark that Atiyah [A10] put bordism and cobordism in the context of generalized homology theories.) For spinor fields the domain spectrum is $M$ Spin or a close variant. The general formula for the anomaly of a spinor field [FH, Conjecture 9.70] - conjectural

\footnotetext{
${ }^{23}$ This is not quite universally true: The anomaly of an $n$-dimensional field theory may not be a full $(n+1)$-dimensional theory, but may only be defined on manifolds of dimension $\leq n$.

${ }^{24}$ More precisely, an $n$-dimensional field theory is a representation of a bordism category $\mathscr{B}$. Following the logic of this paragraph, the anomaly is defined on the same bordism category $\mathscr{B}$, so it is a "once-categorified invertible $n$-dimensional field theory", as indicated in the previous footnote. Typically, the anomaly extends to a full invertible $(n+1)$-dimensional field theory, but that is not required.

${ }^{25}$ This statement is for unitary theories.
} 
as a mathematical assertion until more foundations are laid - brings in the AtiyahBott-Shapiro map $M$ Spin $\rightarrow K O$, as well as all of the aforementioned ingredients. Implicit in it are the various topological and geometric index invariants and index theorems that we have surveyed in this article.

\section{About THE AUthor}

Dan Freed works at the interface of geometry, topology, and physics. He has written many papers on index theory.

\section{REFERENCES}

[A1] M. F. Atiyah, Commentary, Michael Atiyah Collected Works, Index Theory: 1, vol. 3, Oxford University Press, Walton Street, Oxford, 1987, pp. 1-9. 525, 528, 539

[A2] M. F. Atiyah, The index theorem for manifolds with boundary, Seminar on the AtiyahSinger index theorem, With contributions by M. F. Atiyah, A. Borel, E. E. Floyd, R. T. Seeley, W. Shih, and R. Solovay. Annals of Mathematics Studies, No. 57, Princeton University Press, Princeton, N.J., 1965, x+366 pp., Appendix I. 535

[A3] M. F. Atiyah, K-theory and reality, Quart. J. Math. Oxford Ser. (2) 17 (1966), 367-386. 536

[A4] M. F. Atiyah, K-Theory, second ed., Advanced Book Classics, Addison-Wesley, Redwood City, CA, 1989. 538

[A5] M. F. Atiyah, Elliptic operators, discrete groups, and von Neumann algebras, Colloque "Analyse et Topologie" en l'Honneur de Henri Cartan (Orsay, 1974), 1976, pp. 43-72. Astérisque, No. 32-33. 539540

[A6] M. F. Atiyah, Circular symmetry and stationary-phase approximation, Astérisque (1985), no. 131, 43-59. Colloquium in honor of Laurent Schwartz, Vol. 1 (Palaiseau, 1983). 544

[A7] M. F. Atiyah, Elliptic operators and compact groups, Lecture Notes in Mathematics, Vol.

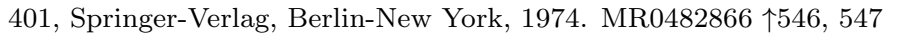

[A8] M. Atiyah, The logarithm of the Dedekind $\eta$-function, Math. Ann. 278 (1987), no. 1-4, 335-380, DOI 10.1007/BF01458075. MR909232 1549

[A9] M. Atiyah, On framings of 3-manifolds, Topology 29 (1990), no. 1, 1-7, DOI 10.1016/0040-9383(90)90021-B. MR1046621 1549

[A10] M. F. Atiyah, Bordism and cobordism, Proc. Cambridge Philos. Soc. 57 (1961), 200-208, DOI 10.1017/s0305004100035064. MR.126856 1520, [560]

[A11] M. F. Atiyah, Topological quantum field theories, Inst. Hautes Études Sci. Publ. Math. (1988), no. 68, 175-186 (1989). 558 559

[A12] M. F. Atiyah, A personal history, The Founders of Index Theory, Reminiscences of Atiyah, Bott, Hirzebruch, and Singer (S. T. Yau, ed.), International Press, 2003, pp. 5-13. 558

[AB1] M. F. Atiyah and R. Bott, A Lefschetz fixed point formula for elliptic complexes. I, Ann. of Math. (2) 86 (1967), 374-407, DOI 10.2307/1970694. MR212836 1532 534542

[AB2] M. F. Atiyah and R. Bott, A Lefschetz fixed point formula for elliptic complexes. II. Applications, Ann. of Math. (2) 88 (1968), 451-491, DOI 10.2307/1970721. MR232406 1535

[AB3] M. F. Atiyah and R. Bott, The index problem for manifolds with boundary, Differential Analysis, Bombay Colloq., 1964, Oxford Univ. Press, London, 1964, pp. 175-186. 535

[AB4] M. Atiyah and R. Bott, On the periodicity theorem for complex vector bundles, Acta Math. 112 (1964), 229-247, DOI 10.1007/BF02391772. MR.178470

[AB5] M. F. Atiyah and R. Bott, The moment map and equivariant cohomology, Topology 23 (1984), no. 1, 1-28, DOI 10.1016/0040-9383(84)90021-1. MR721448 544

[ABP] M. Atiyah, R. Bott, and V. K. Patodi, On the heat equation and the index theorem, Invent. Math. 19 (1973), 279-330, DOI 10.1007/BF01425417. MR650828 1543 544

[ABS] M. F. Atiyah, R. Bott, and A. Shapiro, Clifford modules, Topology 3 (1964), no. suppl, suppl. 1, 3-38, DOI 10.1016/0040-9383(64)90003-5. MR 167985 1524 529 537 538

[ADS] M. F. Atiyah, H. Donnelly, and I. M. Singer, Eta invariants, signature defects of cusps, and values of L-functions, Ann. of Math. (2) 118 (1983), no. 1, 131-177, DOI 10.2307/2006957. MR707164 1547 
[Ag] L. Alvarez-Gaumé, Supersymmetry and the Atiyah-Singer index theorem, Comm. Math. Phys. 90 (1983), no. 2, 161-173. MR714431 1544

[AgG] L. Alvarez-Gaumé and P. Ginsparg, The structure of gauge and gravitational anomalies, Ann. Physics 161 (1985), no. 2, 423-490, DOI 10.1016/0003-4916(85)90087-9. MR793821 1556

[AgW] L. Alvarez-Gaumé and E. Witten, Gravitational anomalies, Nuclear Phys. B 234 (1984), no. 2, 269-330, DOI 10.1016/0550-3213(84)90066-X. MR.736803 1556

[Agr] M. S. Agranovič, Elliptic singular integro-differential operators (Russian), Uspehi Mat. Nauk 20 (1965), no. 5 (125), 3-120. MR.0198017 528

[AH1] M. F. Atiyah and F. Hirzebruch, Vector bundles and homogeneous spaces, Proc. Sympos. Pure Math., Vol. III, American Mathematical Society, Providence, R.I., 1961, pp. 7-38. MR0139181 524

[AH2] M. F. Atiyah and F. Hirzebruch, Riemann-Roch theorems for differentiable manifolds, Bull. Amer. Math. Soc. 65 (1959), 276-281, DOI 10.1090/S0002-9904-1959-10344-X. MR.110106 524

[AHS] M. F. Atiyah, N. J. Hitchin, and I. M. Singer, Self-duality in four-dimensional Riemannian geometry, Proc. Roy. Soc. London Ser. A 362 (1978), no. 1711, 425-461, DOI 10.1098/rspa.1978.0143. MR506229 怆9

[AM] F. Arici and B. Mesland, Toeplitz extensions in noncommutative topology and mathematical physics, arXiv:1911.05823 (2019). 528

[APS1] M. F. Atiyah, V. K. Patodi, and I. M. Singer, Spectral asymmetry and Riemannian geometry. I, Math. Proc. Cambridge Philos. Soc. 77 (1975), 43-69, DOI 10.1017/S0305004100049410. MR397797 1545 546 548 549

[APS2] M. F. Atiyah, V. K. Patodi, and I. M. Singer, Spectral asymmetry and Riemannian geometry. II, Math. Proc. Cambridge Philos. Soc. 78 (1975), no. 3, 405-432, DOI

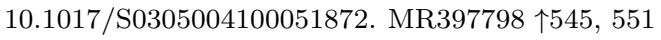

[APS3] M. F. Atiyah, V. K. Patodi, and I. M. Singer, Spectral asymmetry and Riemannian geometry. III, Math. Proc. Cambridge Philos. Soc. 79 (1976), no. 1, 71-99, DOI 10.1017/S0305004100052105. MR397799 1539 545]549]

[AS1] M. F. Atiyah and I. M. Singer, The index of elliptic operators on compact manifolds, Bull. Amer. Math. Soc. 69 (1963), 422-433, DOI 10.1090/S0002-9904-1963-10957-X. MR 1573921528530532

[AS2] M. F. Atiyah and I. M. Singer, The index of elliptic operators. I, Ann. of Math. (2) 87 (1968), 484-530, DOI 10.2307/1970715. MR236950 1529 532 533534

[AS3] M. F. Atiyah and I. M. Singer, The index of elliptic operators. III, Ann. of Math. (2) 87 (1968), 546-604, DOI 10.2307/1970717. MR236952 1526 535

[AS4] M. F. Atiyah and I. M. Singer, The index of elliptic operators. IV, Ann. of Math. (2) 93 (1971), 119-138, DOI 10.2307/1970756. MR279833 1532 538

[AS5] M. F. Atiyah and I. M. Singer, The index of elliptic operators. V, Ann. of Math. (2) 93 (1971), 139-149, DOI 10.2307/1970757. MR279834 1536

[AS6] M. F. Atiyah and I. M. Singer, Index theory for skew-adjoint Fredholm operators, Inst. Hautes Études Sci. Publ. Math. (1969), no. 37, 5-26. 538

[AS7] M. F. Atiyah and I. M. Singer, Dirac operators coupled to vector potentials, Proc. Nat. Acad. Sci. U.S.A. 81 (1984), no. 8, Phys. Sci., 2597-2600, DOI 10.1073/pnas.81.8.2597. $\operatorname{MR} 742394$ 1556

[ASeg] M. F. Atiyah and G. B. Segal, The index of elliptic operators. II, Ann. of Math. (2) 87 (1968), 531-545, DOI 10.2307/1970716. MR236951 t534

[B1] R. Bott, The periodicity theorem for the classical groups and some of its applications, Advances in Math. 4 (1970), 353-411 (1970), DOI 10.1016/0001-8708(70)90030-7. MR259904 1524

[B2] R. Bott, The stable homotopy of the classical groups, Ann. of Math. (2) 70 (1959), 313337, DOI 10.2307/1970106. MR110104 1524

[BdM] L. Boutet de Monvel, On the index of Toeplitz operators of several complex variables, Invent. Math. 50 (1978/79), no. 3, 249-272, DOI 10.1007/BF01410080. MR 520928 1528

[BH2] A. Borel and F. Hirzebruch, Characteristic classes and homogeneous spaces. II, Amer. J. Math. 81 (1959), 315-382, DOI 10.2307/2372747. MR.110105 1524

[BH3] A. Borel and F. Hirzebruch, Characteristic classes and homogeneous spaces. III, Amer. J. Math. 82 (1960), 491-504, DOI 10.2307/2372969. MR.120664 «524 
[Bi1] J.-M. Bismut, The Atiyah-Singer theorems: a probabilistic approach. I. The index theorem, J. Funct. Anal. 57 (1984), no. 1, 56-99, DOI 10.1016/0022-1236(84)90101-0. MR744920 1544

[Bi2] J.-M. Bismut, The Atiyah-Singer index theorem for families of Dirac operators: two heat equation proofs, Invent. Math. 83 (1986), no. 1, 91-151, DOI 10.1007/BF01388755. MR 813584

[BiF1] J.-M. Bismut and D. S. Freed, The analysis of elliptic families. I. Metrics and connections on determinant bundles, Comm. Math. Phys. 106 (1986), no. 1, 159-176. MR.853982 553. 558

[BiF2] J.-M. Bismut and D. S. Freed, The analysis of elliptic families. II. Dirac operators, eta invariants, and the holonomy theorem, Comm. Math. Phys. 107 (1986), no. 1, 103-163. MR861886 553

[BS] A. Borel and J.-P. Serre, Le théorème de Riemann-Roch (French), Bull. Soc. Math. France 86 (1958), 97-136. MR116022 1522

[Bu] U. Bunke, Transgression of the index gerbe, Manuscripta Math. 109 (2002), no. 3, 263287, arXiv:math/0109052 (2001). 558

[BV] N. Berline and M. Vergne, A computation of the equivariant index of the Dirac operator (English, with French summary), Bull. Soc. Math. France 113 (1985), no. 3, 305-345. MR 834043 1545

[BW] R. Brauer and H. Weyl, Spinors in $n$ Dimensions, Amer. J. Math. 57 (1935), no. 2, 425-449, DOI 10.2307/2371218. MR 1507084 1529

[Ca] E. Cartan, Les groupes projectifs qui ne laissent invariante aucune multiplicité plane (French), Bull. Soc. Math. France 41 (1913), 53-96. MR1504700 529

[Ch] C. C. Chevalley, The algebraic theory of spinors, Columbia University Press, New York, 1954. MR0060497 529

[Che] J. Cheeger, $\eta$-invariants, the adiabatic approximation and conical singularities. I. The adiabatic approximation, J. Differential Geom. 26 (1987), no. 1, 175-221. MR892036 1554

[ChS] J. Cheeger and J. Simons, Differential characters and geometric invariants, Geometry and topology (College Park, Md., 1983/84), Lecture Notes in Math., vol. 1167, Springer, Berlin, 1985, pp. 50-80, DOI 10.1007/BFb0075216. MR827262 t550

[Cl] W. K. Clifford, On the classification of geometric algebras, Mathematical Papers (1882), 397-401. 529

[CS] S. S. Chern and J. Simons, Characteristic forms and geometric invariants, Ann. of Math. (2) 99 (1974), 48-69, DOI 10.2307/1971013. MR353327 550

[CZ] A.-P. Calderón and A. Zygmund, Singular integral operators and differential equations, Amer. J. Math. 79 (1957), 901-921, DOI 10.2307/2372441. MR100768 1531

[D] P. A. M. Dirac, The quantum theory of the electron, Proceedings of the Royal Society of London. Series A, Containing Papers of a Mathematical and Physical Character 117 (1928), no. 778, 610-624. 529

[deR] G. de Rham, Sur l'analysis situs des variétés à $n$ dimensions (French), NUMDAM, 1931. MR 3532989 t525

$[\mathrm{DH}]$ J. J. Duistermaat and G. J. Heckman, On the variation in the cohomology of the symplectic form of the reduced phase space, Invent. Math. 69 (1982), no. 2, 259-268, DOI 10.1007/BF01399506. MR674406 1544

[Do] S. K. Donaldson, Atiyah's work on holomorphic vector bundles and gauge theories, Bull. Amer. Math. Soc. 58 (2021), no. 4, 567-610.539 554 556]

[E] C. L. Epstein, Lectures on indices and relative indices on contact and CR-manifolds, Woods Hole mathematics, Ser. Knots Everything, vol. 34, World Sci. Publ., Hackensack, NJ, 2004, pp. 27-93, DOI 10.1142/9789812701398_0002. MR2123367 1528

[F1] D. S. Freed, The Atiyah-Singer index theorem, April 2021. https://www.youtube.com/ watch?v=AJHKp9kYm90. CMSA Math-Science Literature Lecture Series. 518

[F2] D. S. Freed, Classical Chern-Simons theory. II, Houston J. Math. 28 (2002), no. 2, 293310. Special issue for S. S. Chern. 550

[F3] D. S. Freed, Determinants, torsion, and strings, Comm. Math. Phys. 107 (1986), no. 3, 483-513. MR866202 
[FH] D. S. Freed and M. J. Hopkins, Reflection positivity and invertible topological phases, Geom. Topol. 25 (2021), no. 3, 1165-1330, DOI 10.2140/gt.2021.25.1165. MR4268163 1560

[FHT] Daniel S. Freed, Michael J. Hopkins, and Constantin Teleman, Consistent orientation of moduli spaces, The many facets of geometry, Oxford Univ. Press, Oxford, 2010, pp. 395419. arXiv:0711.1909 (2007). 560

[FL] Daniel S. Freed and John Lott, An index theorem in differential K-theory, Geom. Topol. 14 (2010), no. 2, 903-966. arXiv:0907.3508 (2009). 551

[FS] L. D. Faddeev and S. L. Shatashvili, Algebraic and Hamiltonian methods in the theory of nonabelian anomalies (Russian, with English summary), Teoret. Mat. Fiz. 60 (1984), no. 2, 206-217. MR762262 558

[FW] D. Friedan and P. Windey, Supersymmetric derivation of the Atiyah-Singer index and the chiral anomaly, Nuclear Phys. B 235 (1984), no. 3, 395-416, DOI 10.1016/05503213(84)90506-6. MR888706 1544

[G] I. M. Gel'fand, On elliptic equations, Russian Math. Surveys 15 (1960), no. 3, 113-123, DOI 10.1070/RM1960v015n03ABEH004094. MR0123085 †528

[Ge1] E. Getzler, Pseudodifferential operators on supermanifolds and the Atiyah-Singer index theorem, Comm. Math. Phys. 92 (1983), no. 2, 163-178. MR728863 t544

[Ge2] E. Getzler, A short proof of the local Atiyah-Singer index theorem, Topology 25 (1986), no. 1, 111-117, DOI 10.1016/0040-9383(86)90008-X. MR836727 1544

[Gi1] P. B. Gilkey, Curvature and the eigenvalues of the Laplacian for elliptic complexes, Advances in Math. 10 (1973), 344-382, DOI 10.1016/0001-8708(73)90119-9. MR.324731 1543. 544

[Gi2] P. B. Gilkey, The eta invariant for even-dimensional PIN $_{\mathrm{c}}$ manifolds, Adv. in Math. 58 (1985), no. 3, 243-284, DOI 10.1016/0001-8708(85)90119-7. MR815358 551

[GJ] J. Glimm and A. Jaffe, Quantum physics: A functional integral point of view, 2nd ed., Springer-Verlag, New York, 1987, DOI 10.1007/978-1-4612-4728-9. MR887102 1544

[GK] I. C. Gohberg and M. G. Krein, The basic results on the defect numbers, the root numbers and the indices of linear operators (Hungarian), Magyar Tud. Akad. Mat. Fiz. Oszt. Közl. 23 (1977), no. 3-4, 387-460. Translated from the Russian by Károly Buzási. MR 0445320 1528

[H1] F. Hirzebruch, Topological methods in algebraic geometry, Classics in Mathematics, Springer-Verlag, Berlin, 1995. Translated from the German and Appendix One by R. L. E. Schwarzenberger; With a preface to the third English edition by the author and Schwarzenberger; Appendix Two by A. Borel; Reprint of the 1978 edition. MR 1335917 1520

[H2] F. Hirzebruch, A Riemann-Roch theorem for differentiable manifolds, Séminaire Bourbaki, Vol. 5, Soc. Math. France, Paris, 1995, pp. Exp. No. 177, 129-149. 524

[H3] F. Hirzebruch, Some problems on differentiable and complex manifolds, Ann. of Math. (2) 60 (1954) 213-236. 523

[H4] F. E. P. Hirzebruch, Hilbert modular surfaces, Enseign. Math. (2) 19 (1973), 183-281. MR 393045 1546 547

[Hi] N. Hitchin, Harmonic spinors, Advances in Math. 14 (1974), 1-55, DOI 10.1016/00018708(74)90021-8. MR358873

[Ho1] L. Hörmander, Pseudo-differential operators, Comm. Pure Appl. Math. 18 (1965), 501517, DOI 10.1002/cpa.3160180307. MR180740 T535

[Ho2] L. Hörmander, Pseudo-differential operators and hypoelliptic equations, Singular integrals (Proc. Sympos. Pure Math., Vol. X, Chicago, Ill., 1966), 1967, pp. 138-183. 532

[Ho3] L. Hörmander, The analysis of linear partial differential operators. III: Pseudodifferential operators, Grundlehren der Mathematischen Wissenschaften [Fundamental Principles of Mathematical Sciences], vol. 274, Springer-Verlag, Berlin, 1985. MR781536 «532

[Hod] W. V. D. Hodge, Harmonic Functionals in a Riemannian Space, Proc. London Math. Soc. (2) 38 (1935), 72-95, DOI 10.1112/plms/s2-38.1.72. MR1576335 1526

[HS] M. J. Hopkins and I. M. Singer, Quadratic functions in geometry, topology, and M-theory, J. Differential Geom. 70 (2005), no. 3, 329-452. MR2192936 1550

[HZ] F. Hirzebruch and D. Zagier, The Atiyah-Singer theorem and elementary number theory, Mathematics Lecture Series, No. 3, Publish or Perish, Inc., Boston, Mass., 1974. MR 06508321535 
[J] K. Jänich, Vektorraumbündel und der Raum der Fredholm-Operatoren, Math. Ann. 161 (1965), 129-142, DOI 10.1007/BF01360851. MR190946 1538

[JL] J. Jorgenson and S. Lang, Basic analysis of regularized series and products, Lecture Notes in Mathematics, vol. 1564, Springer-Verlag, Berlin, 1993, DOI 10.1007/BFb0077194. MR 1284924 1552

[K] M. Kac, Can one hear the shape of a drum?, Amer. Math. Monthly 73 (1966), no. 4, 1-23, DOI 10.2307/2313748. MR201237 1542

[Kar] M. Karoubi, Algèbres de Clifford et K-théorie (French), Ann. Sci. École Norm. Sup. (4) 1 (1968), 161-270. MR 238927 1538

[KN] J. J. Kohn and L. Nirenberg, An algebra of pseudo-differential operators, Comm. Pure Appl. Math. 18 (1965), 269-305, DOI 10.1002/cpa.3160180121. MR.176362 1532

[L] P. Lévy, Wiener's random function, and other Laplacian random functions, Proceedings of the Second Berkeley Symposium on Mathematical Statistics and Probability, 1950, University of California Press, Berkeley and Los Angeles, 1951, pp. 171-187. MR.0044774 1544

[LM] H. B. Lawson Jr. and M.-L. Michelsohn, Spin geometry, Princeton Mathematical Series, vol. 38, Princeton University Press, Princeton, NJ, 1989. MR1031992 1537

[Lo] Ya. B. Lopatinskiŭ, On a method of reducing boundary problems for a system of differential equations of elliptic type to regular integral equations (Russian), Ukrain. Mat. Ž. 5 (1953), 123-151. MR0073828 535

[Lt1] J. Lott, Higher-degree analogs of the determinant line bundle, Comm. Math. Phys. 230 (2002), no. 1, 41-69,arXiv:math/0106177 (2001). 558

[Lt2] J. Lott, A proof of the axial anomaly, Comm. Math. Phys. 97 (1985), no. 3, 371-379. MR 778621 1556

[Mi1] J. Milnor, On the cobordism ring $\Omega^{*}$ and a complex analogue. I, Amer. J. Math. 82 (1960), 505-521, DOI 10.2307/2372970. MR 119209 1524

[Mi2] J. Milnor, Lectures on the h-cobordism theorem, Princeton University Press, Princeton, N.J., 1965. Notes by L. Siebenmann and J. Sondow. MR0190942 559

[Mik] S. G. Mihlin, Singular integral equations (Russian), Uspehi Matem. Nauk (N.S.) 3 (1948), no. 3(25), 29-112. MR0027429 1531

[MP] S. Minakshisundaram and A. Pleijel, Some properties of the eigenfunctions of the Laplaceoperator on Riemannian manifolds, Canad. J. Math. 1 (1949), 242-256, DOI 10.4153/cjm1949-021-5. MR31145 1541

[MS] H. P. McKean Jr. and I. M. Singer, Curvature and the eigenvalues of the Laplacian, J. Differential Geometry 1 (1967), no. 1, 43-69. MR217739 1542

[Mu W. Müller, Signature defects of cusps of Hilbert modular varieties and values of L-series at $s=1$, J. Differential Geom. 20 (1984), no. 1, 55-119. MR772126 㤷47

[N] F. Noether, Über eine Klasse singulärer Integralgleichungen (German), Math. Ann. 82 (1920), no. 1-2, 42-63, DOI 10.1007/BF01457974. MR 1511970 t528

[NAg] P. Nelson and L. Alvarez-Gaumé, Hamiltonian interpretation of anomalies, Comm. Math. Phys. 99 (1985), no. 1, 103-114. MR.791642 1558

[P1] V. K. Patodi, Curvature and the fundamental solution of the heat operator, J. Indian Math. Soc. 34 (1970), no. 3-4, 269-285 (1971). MR0488181 1543

[P2] V. K. Patodi, An analytic proof of Riemann-Roch-Hirzebruch theorem for Kaehler manifolds, J. Differential Geometry 5 (1971), 251-283. MR290318 1543

[Pa] R. S. Palais, Seminar on the Atiyah-Singer index theorem, Annals of Mathematics Studies, No. 57, Princeton University Press, Princeton, N.J., 1965. With contributions by M. F. Atiyah, A. Borel, E. E. Floyd, R. T. Seeley, W. Shih and R. Solovay. MR0198494 528

[PS] R. S. Palais and R. T. Seeley, Construction of Int ${ }_{k}$, Chapter XVI, Seminar on the AtiyahSinger index theorem, With contributions by M. F. Atiyah, A. Borel, E. E. Floyd, R. T. Seeley, W. Shih and R. Solovay. Annals of Mathematics Studies, No. 57, Princeton University Press, Princeton, N.J., 1965, pp. x+366. 532

[Q] D. Quillen, Determinants of Cauchy-Riemann operators on Riemann surfaces (Russian), Funktsional. Anal. i Prilozhen. 19 (1985), no. 1, 37-41, 96. MR783704 552 553

[Ri] B. Riemann, Theorie der Abel'schen Functionen (German), J. Reine Angew. Math. 54 (1857), 115-155, DOI 10.1515/crll.1857.54.115. MR1579035 1519 
[Ro] G. Roch, Ueber die Anzahl der willkürlichen Constanten in algebraischen Functionen (German), J. Reine Angew. Math. 64 (1865), 372-376, DOI 10.1515/crll.1865.64.372. MR 15793041519

[Roh] V. A. Rohlin, New results in the theory of four-dimensional manifolds (Russian), Doklady Akad. Nauk SSSR (N.S.) 84 (1952), 221-224. MR.0052101 1536

[RS] D. B. Ray and I. M. Singer, R-torsion and the Laplacian on Riemannian manifolds, Advances in Math. 7 (1971), 145-210, DOI 10.1016/0001-8708(71)90045-4. MR295381 1552

[S1] I. M. Singer, 2010. https://www . youtube.com/watch?v=5FoaMcCJnmQ. Interview of Isadore Singer for the MIT+150 Infinite History Project. 525

[S2] I. M. Singer, The $\eta$-invariant and the index, Mathematical aspects of string theory (San Diego, Calif., 1986), Adv. Ser. Math. Phys., vol. 1, World Sci. Publishing, Singapore, 1987, pp. 239-258. 554

[S3] I. M. Singer, Families of Dirac operators with applications to physics, no. Numéro Hors Série, 1985, pp. 323-340. The mathematical heritage of Élie Cartan (Lyon, 1984). 558

[Se1] R. T. Seeley, Elliptic singular integral equations, Singular Integrals (Proc. Sympos. Pure Math., Chicago, Ill., 1966), Amer. Math. Soc., Providence, R.I., 1967, pp. 308-315. MR0234107 1528

[Se2] R. T. Seeley, Singular integrals on compact manifolds, Amer. J. Math. 81 (1959), 658-690, DOI 10.2307/2372921. MR.110022 1532

[Se3] R. T. Seeley, Integro-differential operators on vector bundles, Trans. Amer. Math. Soc. 117 (1965), 167-204, DOI 10.2307/1994203. MR173174 532

[Se4] R. T. Seeley, Complex powers of an elliptic operator, Singular Integrals (Proc. Sympos. Pure Math., Chicago, Ill., 1966), Amer. Math. Soc., Providence, R.I., 1967, pp. 288-307. 541,542

[Seg1] G. Segal, Equivariant K-theory, Inst. Hautes Études Sci. Publ. Math. 34 (1968), 129-151. MR 234452 1534

[Seg2] G. B. Segal, Faddeev's anomaly in Gauss's law. preprint. 558

[Seg3] G. Segal, The definition of conformal field theory, Topology, geometry and quantum field theory, London Math. Soc. Lecture Note Ser., vol. 308, Cambridge Univ. Press, Cambridge, 2004, pp. 421-577. MR2079383 1558

[Seg4] G. B. Segal, Michael Atiyah's work in algebraic topology, Bull. Amer. Math. Soc. 58 (2021), no. 4, 481-516. 519524536

[Sh] H. Shimizu, On discontinuous groups operating on the product of the upper half planes, Ann. of Math. (2) 77 (1963), 33-71, DOI 10.2307/1970201. MR145106 1547

[T1] R. Thom, Quelques propriétés globales des variétés différentiables (French), Comment. Math. Helv. 28 (1954), 17-86, DOI 10.1007/BF02566923. MR61823 1521

[T2] R. Thom, Espaces fibrés en sphères et carrés de Steenrod (French), Ann. Sci. Ecole Norm. Sup. (3) 69 (1952), 109-182. MR0054960 1521

[W] H. Weyl, The classical groups: Their invariants and representations, Princeton Landmarks in Mathematics, Princeton University Press, Princeton, NJ, 1997. Fifteenth printing; Princeton Paperbacks. MR:1488158 1544

[Wi1] E. Witten, Constraints on supersymmetry breaking, Nuclear Phys. B 202 (1982), no. 2, 253-316, DOI 10.1016/0550-3213(82)90071-2. MR668987 1544

[Wi2] E. Witten, Index of Dirac operators, Quantum Fields and Strings: a course for mathematicians, Vol. 1, 2 (Princeton, NJ, 1996/1997), Amer. Math. Soc., Providence, RI, 1999, pp. $475-512.544$

[Wi3] E. Witten, Global gravitational anomalies, Commun. Math. Phys. 100 (1985), 197. 553 554,558

[Wi4] E. Witten, Quantum field theory and the Jones polynomial, Comm. Math. Phys. 121 (1989), no. 3, 351-399. MR990772 1558560

[Wo] R. Wood, Banach algebras and Bott periodicity, Topology 4 (1965/66), 371-389, DOI 10.1016/0040-9383(66)90035-8. MR185598 佔38

Department of Mathematics, University of Texas, Austin, Texas 78712

Email address: dafr@math.utexas.edu 